\title{
THE NUMBER OF TWISTED CUBIC CURVES ON THE GENERAL QUINTIC THREEFOLD*
}

\author{
GEIR ELLINGSRUD and STEIN ARILD STRØMME
}

\begin{abstract}
.
We give explicit descriptions of the Hilbert scheme components parametrizing twisted cubic curves in $\mathbf{P}^{3}$ and $\mathbf{P}^{4}$. As an application, we compute the number 317206375 of twisted cubic curves on a general quintic hypersurface in $\mathbf{P}^{4}$. This coincides with the number predicted by Candelas et. al. in [3].
\end{abstract}

\section{Introduction.}

H. Clemens conjectured in $[5,6]$ that a sufficiently general quintic hypersurface in $\mathbf{P}^{4}$ contains only a finite number of rational curves in each degree. This assertion has been proved by S. Katz [15] for degrees up to 7 . Katz also computed the number 609250 of conics on the general quintic, the number 2875 of lines comes from elementary Schubert calculus. Candelas et al. have a method originating from string theory which predicts the number of rational curves in any degree [3]. The purpose of the present paper is to show that, at least in characteristic zero, the number of twisted cubic curves on the general quintic is 317206375 . Thus we verify the result of the computation of Candelas et. al. for degree three.

For a given degree $d$, let $\mathscr{H}_{d}$ be the irreducible component of the Hilbert scheme of $\mathbf{P}^{4}$ parametrizing rational curves of degree $d$ (and their degenerations). Let $\tilde{\mathscr{C}} \subseteq \mathbf{P}^{4} \times \mathscr{H}_{d}$ be the universal curve, and let $p: \mathbf{P}^{4} \times \mathscr{H}_{d} \rightarrow \mathbf{P}^{4}$ and $\pi: \mathbf{P}^{4} \times \mathscr{H}_{d} \rightarrow \mathscr{H}_{d}$ denote the projections. The short exact sequence

$$
0 \rightarrow \mathscr{I}_{\tilde{\mathscr{E}}} \rightarrow \mathcal{O}_{\mathbf{P}^{4} \times \mathscr{H}_{d}} \rightarrow \mathcal{O}_{\tilde{\mathscr{G}}} \rightarrow 0
$$

gives rise to this exact sequence on $\mathscr{H}_{d}$ :

* Editors note: An unfinished version of this paper was published without the authors' knowledge and consent in the first edition of S.-T. Yau, ed., Essays on Mirror Symmetry, International Press, Hong Kong. The paper will be removed from the new edition of this book.

The second author was supported by the DFG during a stay at Universität Kaiserslautern in May and June 1990.

Received September 27, 1993 


$$
0 \rightarrow \pi_{*} \mathscr{I}_{\tilde{\mathscr{G}}}(5) \rightarrow H^{0}\left(\mathbf{P}^{4}, \mathcal{O}_{\mathbf{P}^{4}}(5)\right)_{\mathscr{H}_{d}} \stackrel{\rho}{\rightarrow} \pi_{*} \mathcal{O}_{\tilde{\mathscr{E}}}(5) \rightarrow R^{1} \pi_{*} \mathscr{I}_{\tilde{\mathscr{G}}}(5) \rightarrow 0 .
$$

Here $\mathscr{I}_{\tilde{\mathscr{G}}}(5)=\mathscr{I}_{\tilde{\mathscr{E}}} \otimes p^{*} \mathcal{O}_{\mathbf{P}^{4}}(5)$ and so on, and the second term denoted the trivial vector bundle on $\mathscr{H}_{d}$ with fiber $H^{0}\left(\mathbf{P}^{4}, \mathcal{O}_{\mathbf{p}^{4}}(5)\right)$. Observe that if $q \in H^{0}\left(\mathbf{P}^{4}, \mathcal{O}_{\mathbf{P}^{4}}(5)\right)$ corresponds to a quintic hypersurface $Q \subseteq \mathbf{P}^{4}$, then the induced section $\rho(q)$ of the coherent sheaf $\pi_{*} \mathcal{O}_{\tilde{\mathscr{E}}}(5)$ vanishes exactly in those points of $\mathscr{H}_{d}$ which correspond to curves contained in $Q$. If $d \leqq 7$, the sheaf $\pi_{*} \mathcal{O}_{\tilde{\mathscr{E}}}(5)$ is locally free. Observe furthermore that the rank of $\pi_{*} \mathcal{O}_{\tilde{\mathscr{E}}}(5)$ equals the dimension $5 d+1$ of $\mathscr{H}_{d}$. This implies, at least in characteristic zero, that whenever $R^{1} \pi_{*} \mathscr{I}_{\tilde{\mathscr{E}}}(5)$ vanishes, the number of rational curves on a general $Q$ is finite, and given by the degree of the top Chern class of the vector bundle $\pi_{*} \mathcal{O}_{\tilde{\mathscr{E}}}(5)$. The vanishing of $R^{1} \pi_{*} \mathscr{I}_{\tilde{\mathscr{E}}}(5)$ is easily verified for $d \leqq 3$. To prove just finiteness, it suffices to show that $R^{1} \pi_{*} \mathscr{I}_{\tilde{\mathscr{E}}}(5)$ vanishes over the open subscheme of $\mathscr{H}_{d}$ which correspond to smooth rational curves. Using [14], one may prove that this is the case for $d \leqq 7$.

If $d=1$, then $\mathscr{H}_{1}$ is the Grassmannian $G(1,4)$ of lines in $\mathbf{P}^{4}$, and $\pi_{*} \mathcal{O}_{\tilde{E}}(5)$ is the 5 -th symmetric power of the canonical rank-2 quotient bundle on $G(1,4)$. The degree of its 6-th Chern class is 2875 . If $d=2$, then $\mathscr{H}_{2}$ is a $\mathbf{P}^{5}$-bundle over the Grassmannian $G(2,4)$ of planes in $\mathbf{P}^{4}$ (since any conic spans a unique plane). One can identify $\pi_{*} \mathcal{O}_{\tilde{\mathscr{E}}}(5)$ in terms of the universal sheaves on the Grassmannian and the projective bundle, and Katz computed that the degree of $c_{11}\left(\pi_{*} \mathcal{O}_{\tilde{\mathscr{E}}}(5)\right)$ is 609250.

From this point of view, computing the number of rational curves of given degree on a general quintic hypersurface depends more on understanding the Hilbert scheme of rational curves in $\mathbf{P}^{4}$ than on the geometry of the quintic threefold. Hence we shall be concerned with the study of twisted cubics and their degenerations. Analogously with the case of conics, any twisted cubic, or flat degeneration thereof, spans a unique hyperplane in $\mathbf{P}^{4}$. Therefore, any reasonable parameter space for these curves will be fibered over the dual space $\mathbf{P}^{4 \vee}$, the fibers being isomorphic to the space of twisted cubics in a fixed $\mathbf{P}^{3}$. The situation is therefore essentially one of codimension two.

To give a rough sketch of the computation, let first $H$ be the irreducible component of the Hilbert scheme of $\mathbf{P}^{3}$ containing the twisted cubic curves. The corresponding curves are of two types: those which are arithmetically Cohen-Macaulay and those that consist of a singular plane cubic plus an embedded point supported in a singularity of the cubic, but not contained in its plane. The latter curves correspond to a Cartier divisor $Y$ on $H$ ( $H$ is nonsingular by [17]), and this divisor clearly admits a fibration $g: Y \rightarrow I$ where $I \subseteq \mathbf{P}^{3} \times \mathbf{P}^{3 \vee}$ is the incidence correspondence $I=\{(x, h) \mid x \in h\}$.

Any curve $C$ corresponding to a point of $H$ determines a net $H^{0}\left(\mathbf{P}^{3}, \mathscr{I}_{C}(2)\right) \subseteq$ $H^{0}\left(\mathbf{P}^{3}, \mathcal{O}_{\mathbf{P}}{ }^{3}(2)\right)$ of quadrics, where $\mathscr{I}_{C}$ denotes the ideal sheaf of $C$. This gives rise to a morphism 


$$
f: H \rightarrow X:=f(H) \subseteq \operatorname{Grass}_{3}\left(H^{0}\left(\mathbf{P}^{3}, \mathcal{O}_{\mathbf{P}^{3}}(2)\right)\right) .
$$

Over $H-Y, f$ is an isomorphism onto its image, and over $Y, f$ is isomorphic to $g$ above, when we identify $I$ with the set of nets of quadrics of the form $\left\langle L_{0}^{2}, L_{0} L_{1}, L_{0} L_{2}\right\rangle$, where the $L_{i}$ are linearly independent linear forms. It turns out that $f$ is isomorphic to the blowup of $X$ along $I$. The structure of the variety $X$ is brought out by exhibiting it as a quotient space for a certain reductive group. In [9] we used this to compute the Chow ring of $X$ in terms of algebra generators and relations. The relevant data on the inclusion of $I$ in $X$ can be computed. With all this we are in position to compute the degree of any given cycle on $H$.

The universal family of curves can be described in the following way. First, note that $X$ inherits a family of nets of quadrics from $\operatorname{Grass}_{3}\left(H^{0}\left(\mathbf{P}^{3}, \mathcal{O}_{\mathbf{P}} 3(2)\right)\right.$. Let $Z \subseteq \mathbf{P}^{3} \times X$ be the zero-scheme of this "universal net of quadrics". Over any point of $X-I$, the fiber of $Z$ is an arithmetically Cohen-Macaulay twisted cubic curve. The fiber of $Z$ over a point on $I$ is just the corresponding plane with an embedded point. Now let $\phi: \tilde{X} \rightarrow X$ be the blowing up of $I$. We show that the inverse image of $Z$ under $\left(\operatorname{id}_{\mathbf{P}^{3}} \times \phi\right)$ has two irreducible components $\tilde{Z}$ and $W$, where $\tilde{Z}$ is the proper transform of $Z$. The component $W$ is easy to describe; it is the pullback of the universal family of planes parametrized by $I$. We show that $\tilde{Z}$ is flat over $\tilde{X}$, and hence induces a morphism $\kappa: \tilde{X} \rightarrow H$ such that $f \circ \kappa=\phi$. It is clear that $\kappa$ is birational and bijective, hence an isomorphism by Zariski's main theorem.

In order to apply this to the problem at hand, we have to rework it when everything happens in a variable hyperplane in $\mathbf{P}^{\mathbf{4}}$. Fortunately, this is quite straightforward; everything mentioned above works in a relative situation, the main difference is that the vector spaces $H^{0}\left(\mathbf{P}^{3}, \mathcal{O}_{\mathbf{P}^{3}}(n)\right)$ are replaced by the symmetric powers of the tautological rank- 4 vector bundle on the base space $\mathbf{P}^{4 \vee}$.

NOTATION AND TERMINOLOGY. All schemes are algebraic over an algebraically closed field $k$ of characteristic 0 . If $T \rightarrow S$ is a morphism of schemes and $\mathscr{F}$ is a coherent sheaf on $S$, we denote its pullback to $T$ by $\mathscr{F}_{T}$.

If $\xi \in A^{1}(S)$ is the rational equivalence class of a Cartier divisor on a scheme $S$, $\mathcal{O}_{S}(\xi)$ will denote the corresponding linebundle. If $T \rightarrow S$ is a morphism, $\mathcal{O}_{T}(\xi)$ denotes the pullback $\mathcal{O}_{S}(\xi)_{T}$.

We use the P of Grotendieck: $\mathbf{P}(\mathscr{F})=\operatorname{Proj}(\operatorname{Symm} \mathscr{F})$. It comes equipped with a linebundle $\mathcal{O}_{\mathbf{P}(\mathscr{F})}(1)$ and a tautological surjection $\mathscr{F}_{\mathbf{P}(\mathscr{F})} \rightarrow \mathcal{O}_{\mathbf{P}(\mathscr{F})}(1)$. When there are several projective bundles around, it is often convenient to specify a name, say $\xi$, for the divisor class $c_{1}\left(\mathcal{O}_{\mathbf{P}(\mathscr{F})}(1)\right) \in A^{1}(\mathbf{P}(\mathscr{F}))$. We express this by saying that $\mathcal{O}_{\mathbf{P}(\mathscr{F})}(\xi)$ is the tautological line bundle on $\mathbf{P}(\mathscr{F})$.

$\operatorname{Grass}^{r}(\mathscr{F})$ is the Grassmannian parametrizing locally free rank-r quotient 
sheaves of $\mathscr{F}$. If $\mathscr{F}$ is locally free, we denote by $\operatorname{Grass}_{r}(\mathscr{F})$ the Grassmannian of rank-r subbundles of $\mathscr{F}$ (canonically isomorphic to $\operatorname{Grass}^{r}\left(\mathscr{F}^{\vee}\right)$ ).

ACKNOWLEDGEMENTs. We want to express our thanks to H. Clemens, W. Fulton, S. Katz, D. Laksov, and D. van Straten for encouragement and stimulating discussions. Special thanks for providing computer facilities go to Universität Kaiserslautern, Bergen Scientific Center, the University of Utah, and the Norwegian Research Council for Science and the Humanities (NAVF).

\section{Twisted cubics.}

A twisted cubic curve $C$ is a smooth, rational, projective curve of degree three. Thus the Hilbert polynomial of $C$ is given as $\chi \mathcal{O}_{C}(v)=3 v+1$. The linear span of such a curve is always three-dimensional, and it is classically known that a twisted cubic is arithmetically normal. This implies that the saturated homogeneous ideal $I_{C}$ of $C$ in its linear span is generated by three quadrics which are the threee $2 \times 2$ minors of a $3 \times 2$ matrix $a$ with linear forms as entries. In particular, there is a resolution

$$
0 \rightarrow 2 \mathcal{O}_{\mathbf{p}} 3(-3) \stackrel{a}{\rightarrow} 3 \mathcal{O}_{\mathbf{p}} 3(-2) \stackrel{\stackrel{2}{A}^{t}}{\longrightarrow} \mathcal{O}_{\mathbf{p}^{3}} \rightarrow \mathcal{O}_{\mathbf{C}} \rightarrow 0 .
$$

Recall [13] that for any projective variety $U$ and any numerical polynomial $\mathbf{P}=P(v)$ there exists a projective scheme $\operatorname{Hilb}_{U}^{P}$ parametrizing all closed subschemes of $U$ with Hilbert polynomial $P$. There exists a universal subscheme $\tilde{Z} \subseteq U \times \operatorname{Hilb}_{U}^{P}$, flat over $\operatorname{Hilb}_{U}^{P}$, such that the fiber over any point $[Z] \in \mathrm{Hilb}_{U}^{P}$ is exactly the subscheme $Z \subseteq U$. Moreover, giving a morphism from a scheme $W$ into Hilb ${ }_{U}^{P}$ is equivalent to specifying a family $Z^{\prime} \subseteq U \times W$, flat over $W$, all the fibers of which have Hilbert polynomial $P$.

Of particular importance to us are the Hilbert schemes Hilb $\mathbf{p}^{3}{ }^{3 v+1}$ and Hilb $\mathbf{p}^{4}{ }^{3 v+1}$. Let us summarize a few basic facts about them. First of all, Hilb $\mathbf{p}^{3 v+1}$ has two irreducible components. One of them contains the set of twisted cubic curves in $\mathbf{P}^{3}$ as a dense open subset. We shall denote this component by $H$. The second irreducible component of $\mathrm{Hilb}_{\mathbf{P}^{3}}^{3 v+1}$ is denoted by $H^{\prime}$. The general point of this component is a smooth plane cubic curve plus an extra point somewhere in $\mathbf{P}^{3}$. It is proved in [17] that $H$ and $H^{\prime}$ are smooth of dimensions 12 and 15 respectively, and that they intersect transversally in a smooth 11-dimensional scheme $Y$. Desnote by $\pi_{H}: \mathbf{P}^{3} \times H \rightarrow H$ the second projection, and let $\tilde{C} \subseteq \mathbf{P}^{3} \times H$ be the universal curve.

There is a clear distinction between the curves in $H-Y$ and those in the divisor $Y$. In fact, they are distinguished by the minimal resolutions:

Lemma 2.1. (i) If $[C] \in H-Y$, then $C$ is arithmetically Cohen-Macaulay, and it has a resolution of the form 


$$
0 \rightarrow 2 \mathcal{O}_{\mathbf{P}^{3}}(-3) \stackrel{a}{\rightarrow} 3 \mathcal{O}_{\mathbf{P}} 3(-2) \stackrel{2}{\wedge^{t} a^{t}} \underset{\mathcal{O}_{\mathbf{P}} 3}{\rightarrow} \mathcal{O}_{\mathbf{C}} \rightarrow 0 .
$$

(ii) If $[C] \in Y$, then $C$ consists of a plane singular cubic curve $C_{0}$ plus an imbedded point in a singularity of $C_{0}$, not contained in the plane of $C_{0}$. If coordinates are chosen such that $x_{0}=0$ and $x_{0}=x_{1}=x_{2}=0$ are the equations for the plane and the point respectively, then the saturated homogeneous ideal $I_{C}$ is given by

$$
I_{C}=\left(x_{0} x_{2}, x_{0} x_{1}, x_{0}^{2}, F\left(x_{1}, x_{2}, x_{3}\right)\right) \text {, }
$$

the equation of $C_{0}$ being $F=a x_{1}^{2}+2 b x_{1} x_{2}+c x_{2}^{2}$ with $a, b$, and $c$ linear forms in $k\left[x_{1}, x_{2}, x_{3}\right]$. Furthermore, a minimal resolution of $\mathcal{O}_{C}$ is of the form

$$
0 \rightarrow \mathcal{O}(-4) \stackrel{\phi}{\rightarrow} 3 \mathcal{O}(-3) \oplus \mathcal{O}(-4) \stackrel{\Psi}{\rightarrow} 3 \mathcal{O}(-2) \oplus \mathcal{O}(-3) \stackrel{\mu}{\rightarrow} \mathcal{O} \rightarrow \mathcal{O}_{C} \rightarrow 0,
$$

where $\mathcal{O}=\mathcal{O}_{\mathbf{p}^{3}}$ and

$\phi=\left(\begin{array}{c}x_{2} \\ x_{1} \\ x_{0} \\ 0\end{array}\right), \quad \psi=\left(\begin{array}{cccc}0 & -x_{0} & x_{1} & c x_{2}+b x_{1} \\ x_{0} & 0 & -x_{2} & b x_{2}+a x_{1} \\ -x_{1} & x_{2} & 0 & 0 \\ 0 & 0 & 0 & -x_{0}\end{array}\right), \quad \mu=\left(x_{0} x_{2}, x_{0} x_{1}, x_{0}^{2}, F\right)$.

Proof: We refer to [17] for most of this. For the last resolution, the only thing which is not obvious is that $\operatorname{Ker} \mu=\operatorname{Im} \psi$. So let $\left(y_{0}, y_{1}, y_{2}, y_{3}\right) \in \operatorname{Ker} \mu$, i.e.,

$$
y_{0} x_{0} x_{2}+y_{1} x_{0} x_{1}+y_{2} x_{0}^{2}+y_{3} F=0 .
$$

Now $x_{0}$ does not divide $F$, so $x_{0} \mid y_{3}$, say $y_{3}=x_{0} y_{3}^{\prime}$. Then we may cancel $x_{0}$ from the equation above to get

$$
\left(y_{0}+y_{3}^{\prime} b x_{1}+y_{3}^{\prime} c x_{2}\right) x_{2}+\left(y_{1}+y_{3}^{\prime} a x_{1}+y_{3}^{\prime} b x_{2}\right) x_{1}+y_{2} x_{0}=0 .
$$

The only relations among $x_{2}, x_{1}$, and $x_{0}$ are the Koszul relations, generated by $\left(x_{1},-x_{2}, 0\right),\left(x_{0}, 0,-x_{2}\right)$, and $\left(0, x_{0},-x_{1}\right)$. Expressing the relation above in terms of these, it is easy to write down an element $\xi$ such that $\psi(\xi)=\left(y_{0}, y_{1}, y_{2}, y_{3}\right)$.

CoROllaRy 2.2. Let $[C] \in H$ be any curve with ideal sheaf $\mathscr{I}_{C}$, and let $v \geqq 1$. Then

$$
H^{1}\left(\mathbf{P}^{3}, \mathscr{I}_{C}(v)\right)=H^{2}\left(\mathbf{P}^{3}, \mathscr{I}_{C}(v)\right)=H^{1}\left(C, \mathcal{O}_{C}(v)\right)=0 .
$$

Furthermore, the sheaves $\pi_{H *} \mathscr{I}_{\tilde{\mathscr{E}}}(v)$ and $\pi_{H *} \mathcal{O}_{\tilde{\mathscr{G}}}(v)$ are locally free of ranks $\left(\begin{array}{c}v+3 \\ 3\end{array}\right)$ $-(3 v+1)$ and $3 v+1$ respectively, and their formation commute with arbitrary base change.

Remarks. (i) Although the curves in $H-Y$ enjoy several properties of the smooth twisted cubics, they may be degenerate. In fact there is a divisor of 
singular curves, the general member of which is the union of a smooth conic and a unisecant line.

(ii) In the lemma above, we may take $a \in k\left[x_{1}, x_{3}\right]$ and $c \in k\left[x_{2}, x_{3}\right]$. Then $a, b$, and $c$ are determined uniquely by the curve $C$.

Now let us turn to Hilb $_{\mathbf{p}^{4}}^{3 v+1}$ which also has two irreducible components. Denote by $\mathscr{H}$ the component containing the twisted cubics. Let $\tilde{\mathscr{C}} \subseteq \mathbf{P}^{4} \times \mathscr{H}$ be the universal curve, and denote by $\mathrm{pr}_{\mathscr{H}}: \mathscr{H} \rightarrow \mathscr{H}$ the second projection.

It is clear that any twisted cubic in $\mathbf{P}^{4}$ is contained in a unique hyperplane. By semicontinuity of the function $[C] \mapsto h^{0}\left(\mathbf{P}^{4}, \mathscr{I}_{C}(1)\right)$, it follows that any curve in $\mathscr{H}$ is contained in a hyperplane. By the classification above, this hyperplane is unique. Thus we may define a map $\Psi: \mathscr{H} \rightarrow \mathbf{P}^{4 \vee}$ by sending [C] to the hyperplane spanned by $C$. Technically, $\mathbf{P}^{4 \vee}$ can be defined as the Grassmannian $S=\operatorname{Grass}^{4}\left(H^{0}\left(\mathbf{P}^{4}, \mathcal{O}_{\mathbf{P}^{4}}(1)\right)\right)$ parametrizing rank-4 quotients of the space of linear forms. Then $S$ carries a universal rank-4 quotient

$$
H^{0}\left(\mathbf{P}^{4}, \mathcal{O}_{\mathbf{p}}(1)\right)_{S} \rightarrow \mathscr{V},
$$

and $\mathbf{P}(\mathscr{V}) \subseteq \mathbf{P}_{S}^{4}$ is the universal hyperplane.

As a matter of notation, let $\mathscr{P}=\mathbf{P}(\mathscr{V}) \stackrel{\pi}{\rightarrow} S$. Let $V$ be a four-dimensional vector space, and put $P=\mathbf{P}(V)$. We denote the tautological linebundle quotients by $V_{P} \rightarrow \mathcal{O}_{P}(1)$ and $\mathscr{V}_{\mathscr{P}} \rightarrow \mathcal{O}_{\mathscr{P}}(1)$ respectively. For any $k$-scheme (resp. $S$-scheme) $T$, we put $P_{T}=P \times T$ (resp. $\left.\mathscr{P}_{T}=\mathscr{P} \times{ }_{S} T\right)$. Let $\pi_{T}: \mathscr{P}_{T} \rightarrow T$ be the natural map. If furthermore $g: T^{\prime} \rightarrow T$ is a morphism, we denote the induced morphism $\mathscr{P}_{T^{\prime}} \rightarrow \mathscr{P}_{T}$ by $\pi^{*} g$. If $\mathscr{F}$ is a coherent sheaf on $\mathscr{P}_{T}$, we may write $\mathscr{F}_{\mathscr{P}_{T}}$ or just $\mathscr{F}_{T^{\prime}}$ for $\left(\pi^{*} g\right)^{*} \mathscr{F}$. Similarly, if $Z \subseteq \mathscr{P}_{T}$ is a subscheme, we write $Z_{T^{\prime}} \subseteq \mathscr{P}_{T^{\prime}}$ for its inverse image under $\pi^{*} g$.

Corollary 2.2 implies that the natural map

$$
H^{0}\left(\mathbf{P}^{4}, \mathcal{O}_{\mathbf{P}^{4}}(1)\right)_{\mathscr{H}} \rightarrow \operatorname{pr}_{\mathscr{*}} \mathcal{O}_{\tilde{\mathscr{E}}}(1)
$$

is a locally free quotient of rank 4 , which by definition of $P^{4 \vee}=S$ is precisely the defining data for a morphism $\Psi: \mathscr{H} \rightarrow S$ such that $\Psi^{*} \mathscr{V}=\operatorname{pr}_{\mathscr{H}_{*}} \mathcal{O}_{\tilde{\mathscr{E}}}(1)$.

LEMMA 2.3. The morphism $\Psi$ so defined is a Zariski locally trivial fibration with fibers isomorphic to $H$. In particular $\mathscr{H}$ is smooth of dimension 16.

Proof. By construction, the universal curve $\tilde{\mathscr{C}}$ is contained in $\mathbf{P}\left(\Psi^{*} \mathscr{V}\right)=$ $\mathscr{P}_{\mathscr{H}} \subseteq \mathbf{P}^{4} \times \mathscr{H}$. Let $U \subseteq S$ be an open subset over which $\mathscr{V}$ is trivial. Then $\mathscr{P}_{U}=\mathbf{P}\left(\left.\mathscr{V}\right|_{U}\right) \simeq \mathbf{P}^{3} \times U$. Furthermore, $\tilde{\mathscr{C}}_{\Psi^{-1}(U)} \simeq \mathscr{P}_{\Psi^{-1}(U)} \simeq \mathbf{P}^{3} \times \Psi^{-1}(U)$ is flat over $\Psi^{-1}(U)$ and hence defines a morphism $\Psi^{-1} U \rightarrow H \times U$, which is easily seen to be an isomorphism.

From this lemma it follows that there is a very close analogy between twisted cubics in $\mathbf{P}^{3}$ and in $\mathbf{P}^{4}$. In fact, we may view the latter as the twisted cubics in some 
"variable" $\mathbf{P}^{3}$, parametrized by the base $S$. We shall often refer to the two cases as the "absolute" and the "relative" case. Notationwise, we shall indicate corresponding objects in the two cases with the same letter, where usually the relative case is indicated by script capitals. Where this is not possible, we shall distinguish the absolute case by a zero subscript.

For example, denote by $\mathscr{Y} \subseteq \mathscr{H}$ the divisor of non-Cohen-Macaulay curves. Then also $\left.\Psi\right|_{y y}: \mathscr{Y} \rightarrow S$ is a Zariski locally trivial fibration with fibers isomorphic to $Y$.

Note also that $\pi_{\mathscr{H}}$ is nothing but the restriction of $\mathrm{pr}_{\mathscr{H}}: \mathbf{P}^{4} \times \mathscr{H} \rightarrow \mathscr{H}$ to the subvariety $\mathscr{P}_{\mathscr{H}}$. In particular, $\pi_{\mathscr{H} *} \mathcal{O}_{\tilde{\mathscr{G}}}(v)=\operatorname{pr}_{\mathscr{H} *} \mathcal{O}_{\tilde{\mathscr{G}}}(v)$ for any $v \in Z$.

As a trivial consequence of corollary 2.2 we have

COROLlaRY 2.4. For any integer $v \geqq 1$, the sheaves $\pi_{\mathscr{H} *} \mathscr{I}_{\tilde{\mathscr{C}}}(v)$ and $\pi_{\mathscr{H} *} \mathcal{O}_{\tilde{\mathscr{E}}}(v)$ are locally free of ranks $\left(\begin{array}{c}v+3 \\ 3\end{array}\right)-(3 v+1)$ and $3 v+1$ respectively, and their formation commute with arbitrary base change. The higher direct images all vanish for $v \geqq 1$.

An important consequence of this is that $\pi_{\mathscr{H} *} \mathcal{O}_{\tilde{\mathscr{E}}}(5)$ is a vector bundle of rank 16 on $\mathscr{H}$, and that the natural map

$$
H^{0}\left(\mathbf{P}^{4}, \mathcal{O}_{\mathbf{P}^{4}}(5)\right)_{\mathscr{H}} \stackrel{\rho}{\rightarrow} \pi_{\mathscr{H} *} \mathcal{O}_{\tilde{\mathscr{E}}}(5)
$$

is surjective. Let $F \in H^{0}\left(\mathbf{P}^{4}, \mathcal{O}_{\mathbf{p}}(5)\right)$ be a homogeneous quintic polynomial. Then $F$ induces a global section $\rho(F)$ of $\pi_{\mathscr{H} *} \mathcal{O}_{\tilde{\mathscr{E}}}(5)$. If $[C] \in \mathscr{H}$ is a point, the fiber of $\pi_{\mathscr{H} *} \mathcal{O}_{\tilde{\mathscr{G}}}(5)$ in $[C]$ is just $H^{0}\left(C, \mathcal{O}_{\mathrm{C}}(5)\right)$, and the value of $\rho(F)$ on this fiber is nothing but the restriction of $F$ to $C$. Hence $\rho(F)$ vanishes at $[C]$ if and only if $C$ is contained in $V(F)$, the quintic hypersurface defined by $F$.

PROPOSITION 2.5. For a general quintic form $F$, the number of twisted cubics on $V(F) \subseteq \mathbf{P}^{4}$ is given by the degree $\int_{\mathscr{H}} c_{16}\left(\pi_{\mathscr{H} *} \mathcal{O}_{\tilde{\mathscr{G}}}(5)\right)$. These cubics are all smooth.

Remark. One may show that the normal bundle of each of these twisted cubic splits in a sum of two linebundles of degree -1 . Cfr. [15].

ProOF. Since the map in (2-2) is surjective, the vector bundle $\pi_{\mathscr{H} *} \mathcal{O}_{\tilde{\mathscr{E}}}(5)$ is generated by the space $H^{0}\left(\mathbf{P}^{4}, \mathcal{O}_{\mathbf{P}}(5)\right)$ of global sections. In characteristic zero, a general such section is transverse to the zero section, meaning that it vanishes in a smooth subscheme of codimension 16 , hence a finite number of reduced points. The section can be chosen to have zeroes outside of any given proper closed subset, like the locus of singular curves, for example. It is well known that the number of zeroes of such a section is the degree of the top Chern class of the vector bundle.

REMARK. With this proposition, we have translated the problem of counting 
the cubics on the general quintic from a problem on quintic threefolds to a problem of the enumerative geometry of the cubics. What remains is to determine the cohomology ring of $\mathscr{H}$ and give a description of $\pi_{\mathscr{H} *} \mathcal{O}_{\tilde{\mathscr{E}}}(5)$ suitable for computing the degree of the top Chern class. We shall not see more of the quintic itself in this paper.

\section{Determinantal nets of quadrics.}

Any curve $C$ in $H$ is contained in a net (i.e., three-dimensional vector space, or two-dimensional projective space) of quadrics. These nets are all determinantal, meaning that they are generated by the $2 \times 2$ minors of a $3 \times 2$ matrix of linear forms. For the curves in $H-Y$, this follows immediately from lemma 2.1 (i). For curves in $Y$, the matrix

$$
\left(\begin{array}{cc}
0 & -\mathrm{x}_{0} \\
\mathrm{x}_{0} & 0 \\
-\mathrm{x}_{1} & \mathrm{x}_{2}
\end{array}\right)
$$

will do, where the coordinates are chosen as in lemma 2.1 (ii).

Recall that $V=H^{0}\left(\mathbf{P}^{3}, \mathcal{O}_{\mathbf{P}^{3}}(1)\right)$. Let $S_{n} V=H^{0}\left(\mathbf{P}^{3}, \mathcal{O}_{\mathbf{P}^{3}}(n)\right)$ denote its $n$th symmetric product. From corollary 2.2, the following exact sequence on $H$

$$
0 \rightarrow \pi_{H *} \mathscr{I}_{\tilde{\mathscr{G}}}(2) \rightarrow S_{2} V_{H} \rightarrow \pi_{H *} \mathcal{O}_{\tilde{\mathscr{G}}}(2) \rightarrow 0
$$

gives a morphism $f_{0}: H \rightarrow \operatorname{Grass}_{3}\left(S_{2} V\right)$ associating to $[C]$ the net of quadrics containing it. Put $X=f_{0}(H)$ and equip it with the reduced subscheme structure. Similarly, put $I=f_{0}(Y)$, again with the reduced subscheme structure.

A preliminary analysis of the morphism $f_{0}$ reveals that it induces a bijection $H-Y \rightarrow X-I$, since an arithmetically Cohen-Macaulay curve is cut out by its net of quadrics. For a curve $C$ in $Y$, however, the net of quadrics has a linear common factor, and remembers only the plane spanned by the cubic $C_{0}$ and the location of the imbedded point; the plane curve $C_{0}$ itself is lost. Hence the points of $I$ are in bijection with the incidence correspondence in $\mathbf{P}^{3} \times \mathbf{P}^{3 \vee}$, i.e., $\{(p, q) \mid p$ a point, $q$ a plane, $p \in q\}$. The fiber of $f_{0}$ over such a pair $(p, q)$ is the space of all cubics in the plane $q$ passing doubly through the point $p$, hence the fiber is isomorphic to $\mathbf{P}^{6}$. We are going to show later that $f_{0}$ is the blowing up of $X$ with center $I$.

In the relative situation, it follows from corollary 2.4 that the exact sequence on $\mathscr{H}$

$$
0 \rightarrow \pi_{\mathscr{H} *} \mathscr{I}_{\tilde{\mathscr{E}}}(2) \rightarrow \mathrm{S}_{2} \mathscr{V}_{\mathscr{H}} \rightarrow \pi_{\mathscr{H} *} \mathcal{O}_{\tilde{\mathscr{E}}}(2) \rightarrow 0
$$

defines an $S$-morphism $f: \mathscr{H} \rightarrow \operatorname{Grass}_{3}\left(S_{2} \mathscr{V}\right)$. It is clear that the fiber of $f$ over any point in $S$ is just $f_{0}$. Let $\mathscr{X} \subseteq \operatorname{Grass}_{3}\left(S_{2} \mathscr{V}\right)$ be the image of $f$ and $\mathscr{I} \subseteq \mathscr{X}$ the 
image of $\mathscr{Y}$ under $f$, again with their reduced subscheme structures. Then $\mathscr{X} \rightarrow S$ and $\mathscr{Y} \rightarrow S$ and $\mathscr{I} \rightarrow S$ are all Zariski locally trivial fibrations with fibers $X, Y$, and $I$ respectively.

The space $X$ was introduced in [10] where some of its basic properties were proved. We shall quickly review some of these. The key to proving these properties is an alternative description of the space $X$, namely as the quotient of the space of $3 \times 2$ matrices of linear forms by the natural action of the group $\mathrm{GL}(3) \times \mathrm{GL}(2)$.

To give this description, let $E$ and $F$ be two vector spaces of dimensions 3 and 2 , respectively. Let $W=\operatorname{Hom}_{k}(F, E \otimes V)$. Having chosen bases for $E$ and $F$, the elements of $W$ may be interpreted as $3 \times 2$ matrices with entries in $V$.

Let $\alpha$ be an element of $W$. The subspace of $S_{2} V$ generated by the maximal minors of a matrix representing $\alpha$ does not depend on the choice of the matrix. We shall denote this subspace by $E(\alpha)$.

The group $\mathrm{GL}(E) \times \mathrm{GL}(F)$ acts naturally on $W$ by

$$
(g, h) \cdot \alpha=\left(g \otimes 1_{V}\right) \circ \alpha \circ h^{-1} .
$$

The normal subgroup $\Gamma=\left\{\left(t \mathrm{id}_{E}, t \mathrm{id}_{F}\right) \mid t \in k^{*}\right\}$ acts trivially, and thus the quotient $G=\mathrm{GL}(E) \times \mathrm{GL}(F) / \Gamma$ acts on $W$.

We call an element $\alpha \in W$ stable (resp. semistable) if the corresponding point $\bar{\alpha} \in \mathbf{P}\left(W^{\vee}\right)$ is stable (resp. semistable) in the sense of Mumford [16] for the induced action of $G \cap \operatorname{SL}(W)$.

Proposition 3.1. For any element $\alpha \in W$, the following are equivalent:

(i) $\alpha$ is stable.

(ii) $\alpha$ is semistable.

(iii) $\operatorname{dim} E(\alpha)=3$.

(iv) For no choice of bases for $E$ and $F$ will the corresponding $3 \times 2$ matrix have two zeroes in one column or in one row.

Proof. See [10, lemma 1].

RemarK. This is a special case of a more general result by Drezet [8].

The equivalence of (i) and (ii) implies by [16] that there exists a projective, geometric quotient $W^{s} / G$, and from (iii) it follows that there is a morphism $W^{s} / G \rightarrow X$.

Proposition 3.2. (i) The action of $G$ on $W^{s}$ is free, hence $W^{s} / G$ is smooth.

(ii) The morphism $W^{s} / G \rightarrow X$ is an isomorphism.

ProOF. See [10, prop.1].

On $W^{s} / G$ there are two bundles $\mathscr{F}^{\prime}$ and $\mathscr{E}^{\prime}$ which are fundamental for what we 
are going to do. They are obtained by descending the $G$-bundles $E_{W^{s}}$ and $F_{W^{s}}$ to $W^{s} / G$. To obtain $G$-actions on $E$ and $F$, let $\operatorname{GL}(E) \times \mathrm{GL}(F)$ act by

$$
\begin{aligned}
& (g, h) \cdot e=\operatorname{det}(h) \operatorname{det}(g)^{-1} g(e) \quad(e \in E) \\
& (g, h) \cdot f=\operatorname{det}(h) \operatorname{det}(g)^{-1} h(f) \quad(f \in F) .
\end{aligned}
$$

The induced actions of $\Gamma$ are trivial, hence $G$ acts on $E$ and $F$, so these descend to vector bundles $\mathscr{E}^{\prime}$ and $\mathscr{F}^{\prime}$ on $W^{s} / G$. The determinants of the two representations (3-1) are represented by the same character of $G$. It follows that $\operatorname{det}\left(\mathscr{E}^{\prime}\right)=\operatorname{det}\left(\mathscr{F}^{\prime}\right)$. The tautological map $F_{W} \rightarrow E_{W} \otimes V$ is $G$-equivariant, hence descends to a bundle $\operatorname{map} A^{\prime}: \mathscr{F}^{\prime}-\mathscr{E}^{\prime} \otimes V$ on $W^{s} / G$.

The bundles $\mathscr{E}^{\prime}$ and $\mathscr{F}^{\prime}$ may be described also in another way. Let $\mathscr{E}_{0} \subseteq S_{2} V$ be the restriction to $X$ of the canonical rank-3 subbundle on $\operatorname{Grass}_{3}\left(S_{2} V\right)$. The multiplication map $S_{2} V \otimes V \rightarrow S_{3} V$ induces a map $\mathscr{E}_{0} \otimes V \rightarrow S_{3} V_{X}$ whose kernel we denote by $\mathscr{F}_{0}$. It is easily checked, using that the nets in $X$ are determinantal, that $\mathscr{F}_{0}$ is a vector bundle of rank two. Let $A: \mathscr{F}_{0} \rightarrow \mathscr{E}_{0} \otimes V$ be the inclusion map.

Proposition 3.3. Under the isomorphism $W^{s} / G \rightarrow X$, the bundles $\mathscr{E}^{\prime}$ and $\mathscr{F}^{\prime}$ correspond to the bundles $\mathscr{E}_{0}$ and $\mathscr{F}_{0}$, and the map $A^{\prime}$ corresponds to the map $A$.

Proof. Consider the map $\alpha: \pi_{W^{s} / G^{*}}^{\mathscr{F}^{\prime}} \rightarrow \pi_{W^{s} / G}^{*} \mathscr{E}^{\prime}(1)$ on $P_{W^{s} / G}$. The EagonNorthcott complex on $\alpha$ is

$$
0 \rightarrow \pi_{W^{s} / G}^{*} \mathscr{F}^{\prime} \stackrel{\alpha}{\rightarrow} \pi_{W^{s} / G}^{*} \mathscr{E}^{\prime}(1) \stackrel{2}{\wedge} \alpha^{t} \rightarrow \pi_{W^{s} / G}^{*}\left(\operatorname{det}\left(\mathscr{F}^{\prime}\right)^{-1} \otimes \operatorname{det}\left(\mathscr{E}^{\prime}\right)\right)(3)
$$

Untwisting by 1 and applying $\pi_{W^{s} / G *}$ yields a map $\mathscr{E}^{\prime} \rightarrow S_{2} V$, since $\operatorname{det}\left(\mathscr{E}^{\prime}\right)=\operatorname{det}\left(\mathscr{F}^{\prime}\right)$. The rest is easy.

To globalize the two bundles above to the relative case, let $\mathscr{E}$ be the restriction to $\mathscr{X}$ of the tautological subbundle of $S_{2} \mathscr{V}_{\mathrm{Grass}_{3}\left(\mathrm{~S}_{2} \mathscr{V}\right)}$, and let $\mathscr{F}$ be the kernel of the map $\mathscr{E} \otimes \mathscr{V} \rightarrow S_{3} \mathscr{V}$ induced by multiplication. Also, let $\mathscr{A}: \mathscr{F} \rightarrow \mathscr{E} \otimes \mathscr{V}$ be the inclusion map. Then clearly the bundles $\mathscr{E}$ and $\mathscr{F}$ restrict to $\mathscr{E}_{0}$ and $\mathscr{F}_{0}$ on each closed fiber over $S$, and $\mathscr{A}$ restricts to $A$.

Composing the "tautological" map $\mathscr{A}$ with the natural surjection $\mathscr{V}_{\mathscr{P}} \rightarrow \mathcal{O}_{\mathscr{P}}(1)$, we obtain the following map on $\mathscr{P}_{\mathscr{X}}$ :

$$
\text { a: } \pi_{\mathscr{X}}^{* \mathscr{F}} \rightarrow \pi_{\mathscr{X}}^{*} \mathscr{E} \otimes \mathcal{O}_{\mathscr{P}_{\mathscr{X}}}(1) .
$$

The degeneration locus of this bundle map is denoted by $\mathscr{Z} \subseteq \mathscr{P}_{\mathscr{X}}$, i.e., $\mathscr{Z}$ is the locus where $a$ has rank at most one. It is locally defined by the maximal minors of $a$, and hence we may build the Eagon-Northcott complex on $a$ :

$$
0 \rightarrow \pi_{\mathscr{X}}^{*} \mathscr{F}(-3) \stackrel{a}{\rightarrow} \pi_{\mathscr{X}}^{*} \mathscr{E}(-2) \stackrel{2^{2} a^{t}}{\longrightarrow} \mathcal{O}_{\mathscr{X}_{\mathscr{X}}} \rightarrow \mathcal{O}_{\mathscr{X}} \rightarrow 0 .
$$

Recall that any irreducible component of $\mathscr{Z}$ is of codimension at most two, and that (3-2) is exact if and only if $\mathscr{Z}$ is of pure codimension two. Let $x \in \mathscr{X}-\mathscr{I}$. 
Then the minors of $a(x)$ define a curve, hence the inverse image of $\mathscr{X}-\mathscr{I}$ in $\mathscr{Z}$ is of codimension two. Since $\mathscr{I} \subseteq \mathscr{X}$ is of codimension seven, the inverse image of $\mathscr{I}$ in $\mathscr{Z}$ has codimension at least four, hence it cannot be an irreducible component. It follows that $\mathscr{Z}$ is irreducible of codimension two, and the complex (3-2) is exact.

Let us also remark that if $x \in \mathscr{X}-\mathscr{I}$, then the fiber of (3-2) over $x$ is, up to the choice of bases for $E$ and $F$, nothing but the resolution (2-1). In particular, $\mathscr{Z}_{\mathscr{X}-\mathscr{I}}$ is flat over $\mathscr{X}-\mathscr{I}$, and we obtain a morphism $\mathscr{X}-\mathscr{I} \rightarrow \mathscr{H}-f^{-1}(\mathscr{I})$ which almost by definition is an inverse to $\left.f\right|_{\mathscr{H}-y}$. Hence we have proven

Proposition 3.4. $f: \mathscr{H} \rightarrow \mathscr{X}$ induces an isomorphism $\left.f\right|_{\mathscr{H}-\mathscr{y}:}: \mathscr{H}-\mathscr{Y} \stackrel{\approx}{\longrightarrow}$ $\mathscr{X}-\mathscr{I}$. Under this isomorphism, $\mathscr{Z}_{\mathscr{X}-\mathscr{I}}$ corresponds to $\mathscr{C}_{\mathscr{H}-\mathscr{y}}$.

REMARK. There is of course a similar statement in the absolute case. Later we will show that $f$ is the blowing up of $\mathscr{X}$ along $\mathscr{I}$, see cor. 5.4.

\section{The degenerate nets.}

We are going to study the inclusion of $I$ into $X$ and its parallel in the relative case. We do the absolute case first; then the generalization to the relative case will be straightforward. The main objective is to compute the normal bunle of $\mathscr{I}$ in $\mathscr{X}$.

It is convenient at this point to redefine $I$ to be the incidence correspondence in $P \times P^{\vee}$ and then define a certain morphism $i_{0}: I \rightarrow X$, which in turn we will prove to be a closed imbedding onto the subscheme $f_{0}(Y)$ of $X$ (earlier denoted by $I$ ). Once we have done this, we identify $I$ with its image in $X$, thus reverting to the old definition.

So let $I \subseteq P \times P^{\vee}$ be defined in the following way: Recall that $P=\mathbf{P}(V)$, and of course $P^{\vee}=\mathbf{P}\left(V^{\vee}\right)$. There will soon be many tautological linebundles, so we start naming them: Let $\lambda: V_{P} \rightarrow \mathcal{O}_{P}(\xi)$ and $\mu: V_{P^{\vee}}^{\vee} \rightarrow \mathcal{O}_{P^{\vee}}\left(\xi^{\vee}\right)$ be the tautological quotients on $P$ and $P^{\vee}$ respectively. (Of course, the tautological linebundle on $P$ is $\mathcal{O}_{P}(1)$. We introduce this notation to distinguish two different copies of $P$ which enter into later calculations.) Then $I$ is defined as the zero-scheme of the composed map on $P \times P^{\vee}$ :

$$
\mathcal{O}_{\boldsymbol{P} \times \boldsymbol{P}^{\vee}}\left(-\xi^{\vee}\right) \stackrel{\mu^{t}}{\longrightarrow} V_{\boldsymbol{P} \times \boldsymbol{P}^{\vee}} \stackrel{\lambda}{\longrightarrow} \mathcal{O}_{\boldsymbol{P} \times \boldsymbol{P}^{\vee}}(\xi) .
$$

The characteristic data of $I$ are summarized in the diagram 


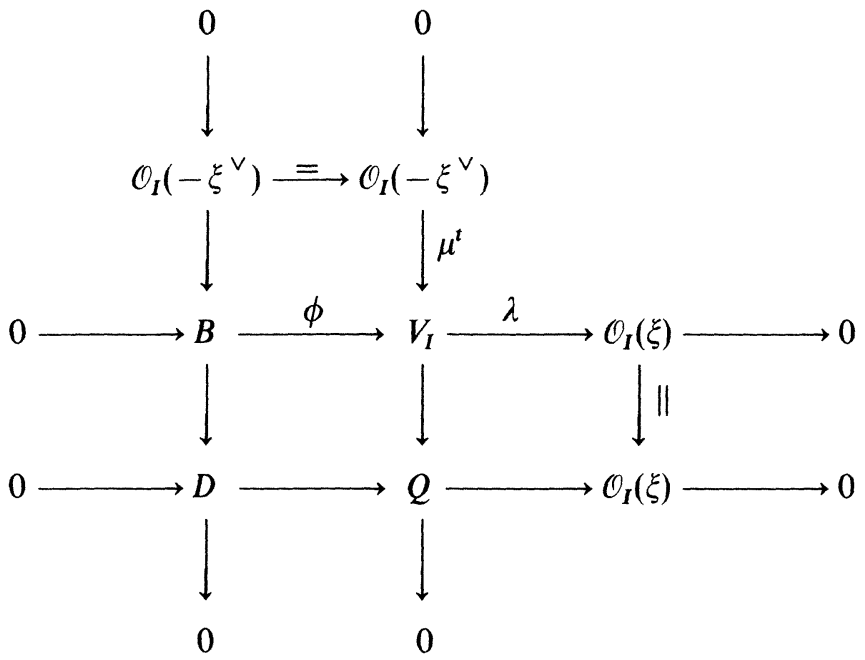

which defines the rank-3 bundles $B$ and $Q$ and the rank- 2 bundle $D$ on $I$. We shall refer to $\mathbf{P}(Q) \subseteq \mathbf{P}\left(V_{I}\right)=\mathrm{P}_{I}$ as the "universal plane" and to $\Sigma_{0}=\mathbf{P}\left(\mathcal{O}_{P}(\xi)\right) \subseteq P_{I}$ as the "universal point". Clearly, $\Sigma_{0} \subseteq \mathbf{P}(Q)$, and $\Sigma_{0} \rightarrow I$ is an isomorphism.

Now let a point $x \in I$ be given. Choose coordinates $x_{0}, x_{1}, x_{2}, x_{3} \in V$ such that $x_{0}=0$ is the equation for the plane and $x_{0}=x_{1}=x_{2}=0$ are the equations for the point. Then $\phi(B) \otimes k(x)=\left\langle x_{0}, x_{1}, x_{2}\right\rangle$ and $\mu^{t}\left(\mathcal{O}_{I}\left(-\xi^{\vee}\right)\right) \otimes k(x)=\left\langle x_{0}\right\rangle$.

To construct the map $i_{0}: I \rightarrow X$, we shall give a rank-3 subbundle of $S_{2} V_{I}$. Clearly the image of the composition of $\mu^{t} \otimes \phi: \mathcal{O}_{I}\left(-\xi^{\vee}\right) \otimes B \rightarrow V_{I} \otimes V_{I}$ with the multiplication map $V_{I} \otimes V_{I} \rightarrow S_{2} V_{I}$ is a rank-3 subbundle which at the point $x$ has fiber $\left\langle x_{0} x_{2}, x_{0} x_{1}, x_{0}^{2}\right\rangle$. This defines a morphism $i_{0}: I \rightarrow \operatorname{Grass}_{3}\left(S_{2} V\right)$, easily seen to factor through $X$. This is a bijection onto $f_{0}(Y)$. Now GL(V) operates naturally on $I$ and $X$, and the morphism $i_{0}$ is equivariant. Since the action is transitive on $I$, it follows that $i_{0}$ is a closed imbedding onto $f_{0}(Y)$.

From now on, we identify $I$ with $f_{0}(Y)$ via $i_{0}$, thus resuming our earlier definition.

It is straightforward how this construction generalizes to the relative situation. Let $\mathscr{P}=\mathbf{P}(\mathscr{V}) \rightarrow S$ be as before and put $\mathscr{P}^{\vee}=\mathbf{P}\left(\mathscr{V}^{\vee}\right) \rightarrow S$. Again we denote by $\lambda: \mathscr{V}_{\mathscr{P}} \rightarrow \mathcal{O}_{\mathscr{P}}(\xi)$ and $\mu: \mathscr{V}_{\mathscr{P}^{\vee}}^{\vee} \rightarrow \mathcal{O}_{\mathscr{P}^{\vee}}\left(\xi^{\vee}\right)$ the tautological quotients on $\mathscr{P}$ and $\mathscr{P}^{\vee}$ respectively. Then $\mathscr{I}$ is defined as the zero-scheme of the composed map on $\mathscr{P} \times{ }_{S} \mathscr{P}^{\vee}$ :

$$
\mathcal{O}_{\mathscr{P} \times s} \mathscr{P}^{\vee}\left(-\xi^{\vee}\right) \stackrel{\mu^{t}}{\longrightarrow} \mathscr{V}_{\mathscr{P} \times_{s} \mathscr{P}^{\vee}} \stackrel{\lambda}{\longrightarrow} \mathcal{O}_{\mathscr{P} \times \mathfrak{P}^{\vee}}(\xi)
$$

The characteristic data of $\mathscr{I}$ are summarized in the exact commutative diagram 
$(4-1)$

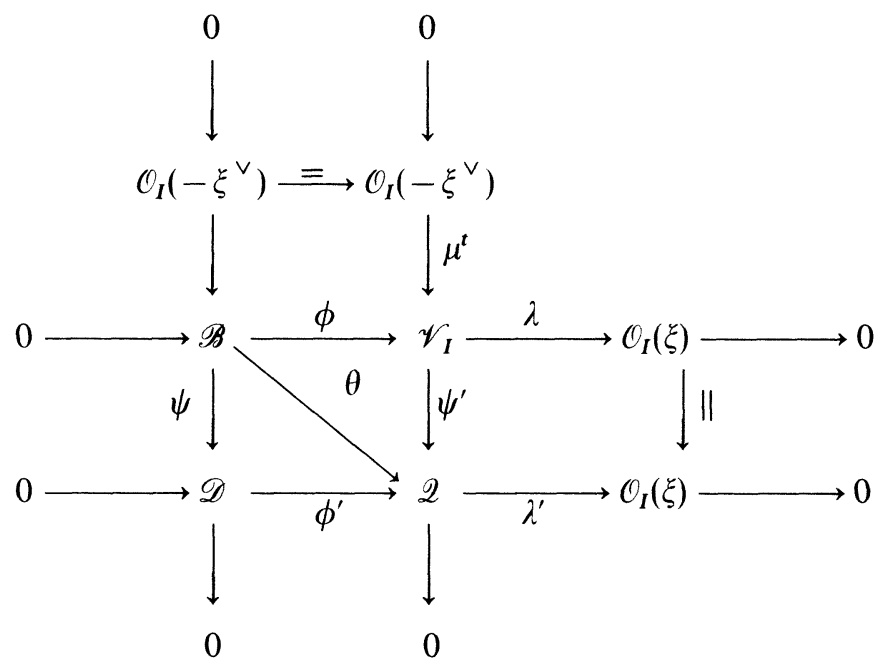

defining rank- 3 bundles $\mathscr{B}$ and $\mathscr{Q}$ and a rank-2 bundle $\mathscr{D}$ on $\mathscr{I}$. Clearly $\mathscr{I} \rightarrow S$ is a Zariski locally trivial fibration with fiber $I$, and the bundles on $\mathscr{I}$ restrict on the fibers to their unscripted counterparts. In absolute terms, a point of $\mathscr{I}$ consists of a triple $(p, q, s)$ where $s$ is a hyperplane in $\mathbf{P}^{4}, q$ a hyperplane in $s$, and $p$ a point of $q$. The morphism $\mathscr{I} \rightarrow S$ is given by $(p, q, s) \mapsto s$.

We refer to $\mathbf{P}(\mathscr{Q}) \subseteq \mathbf{P}\left(\mathscr{V}_{I}\right)=\mathscr{P}_{\mathscr{I}}$ as the "universal plane" and to $\Sigma=\mathbf{P}\left(\mathcal{O}_{\mathscr{P}}(\xi)\right) \subseteq \mathscr{P}_{\mathscr{F}}$ as the "universal point". Clearly, $\Sigma \subseteq \mathbf{P}(\mathscr{Q})$, and $\Sigma \rightarrow \mathscr{I}$ is an isomorphism.

We proceed to construct the map $i: \mathscr{I} \rightarrow \mathscr{X}$. In complete analogy with the absolute case, we get the required rank- 3 subbundle of $S_{2} \mathscr{V}_{\mathscr{I}}$ by taking the image of the composition

$$
\mathcal{O}_{\mathscr{F}}\left(-\xi^{\vee}\right) \otimes \mathscr{B} \stackrel{\mu^{\prime} \otimes \phi}{\longrightarrow} \mathscr{V}_{\mathscr{I}} \otimes \mathscr{V}_{\mathscr{I}} \stackrel{\text { mult }}{\longrightarrow} S_{2} \mathscr{V}_{\mathscr{I}} .
$$

By construction, this gives the morphism $i_{0}$ on each fiber over $S$, and we conclude that $i: \mathscr{I} \rightarrow \mathscr{X}$ is an isomorphism onto $f(\mathscr{Y})$. Henceforth we shall identify $\mathscr{I}$ with $f(\mathscr{Y})$ via $i$.

The next step is to identify the restrictions of $\mathscr{E}$ and $\mathscr{F}$ to $\mathscr{I}$.

Lemma 4.1. (i) We have $i^{*} \mathscr{E}=\mathscr{B}\left(-\xi^{\vee}\right)$ and $i^{*} \mathscr{F}=\mathscr{D}^{*}\left(-\xi^{\vee}\right) \otimes \operatorname{det}(\mathscr{B})$.

(ii) If $\mathscr{A} \in \operatorname{Hom}\left(\mathscr{F}, \mathscr{E} \otimes \mathscr{V}_{\mathscr{X}}\right)$ is the tautological map, then $i^{*} \mathscr{A} \in \operatorname{Hom}\left(i^{*} \mathscr{F}\right.$, $\left.i^{*} \mathscr{E} \otimes \mathscr{V}_{\mathscr{I}}\right)=H^{\circ}\left(\mathscr{I}, \operatorname{det}(\mathscr{B})^{-1} \otimes \mathscr{D} \otimes \mathscr{B} \otimes \mathscr{V}_{\mathscr{I}}\right)=\operatorname{Hom}\left(\operatorname{det}(\mathscr{B}) . \quad \mathscr{D} \otimes \mathscr{B} \otimes \mathscr{V}_{\mathscr{I}}\right)$ is given by the composition

$$
\operatorname{det}(\mathscr{B})=\stackrel{3}{\wedge} \mathscr{B} \stackrel{r}{\rightarrow} \mathscr{B} \otimes \mathscr{B} \otimes \mathscr{B} \stackrel{\psi \otimes 1 \otimes \phi}{\longrightarrow} \mathscr{D} \otimes \mathscr{B} \otimes \mathscr{V}_{\mathscr{F}},
$$

where $r$ is the natural polarization map 


$$
\beta_{0} \wedge \beta_{1} \wedge \beta_{2} \mapsto \sum_{\sigma \in S_{3}} \operatorname{sign}(\sigma) \beta_{\sigma(0)} \otimes \beta_{\sigma(1)} \otimes \beta_{\sigma(2)} .
$$

In other words, $i^{*} \mathscr{A}$ is locally given by

$$
\begin{gathered}
\wedge \stackrel{B}{\mathscr{B}} \rightarrow \mathscr{D} \otimes \mathscr{B} \otimes \mathscr{V}_{\mathscr{G}} \\
\beta_{0} \wedge \beta_{1} \wedge \beta_{2} \mapsto \sum_{\sigma \in S_{3}} \operatorname{sign}(\sigma) \psi \beta_{\sigma(0)} \otimes \beta_{\sigma(1)} \otimes \beta_{\sigma(2)} .
\end{gathered}
$$

ProOF. That $i^{*} \mathscr{E}=\mathscr{B}\left(-\xi^{\vee}\right)$ follows from (4-2) and the definition of $i$. For the rest, let

$$
\alpha: \mathscr{D}^{*} \otimes \operatorname{det}(\mathscr{B}) \rightarrow \mathscr{B} \otimes \mathscr{V}_{\mathscr{I}}
$$

be the map induced by (4-3). We need to show that $\alpha$ is pointwise injective and that the composition

$$
\begin{aligned}
\mathcal{O}_{\mathscr{F}}\left(-\xi^{\vee}\right) \otimes \mathscr{D}^{*} \otimes \operatorname{det}(\mathscr{B}) \stackrel{1 \otimes \alpha}{\longrightarrow} \mathcal{O}_{\mathscr{F}}\left(-\xi^{\vee}\right) \otimes \mathscr{B} \otimes \mathscr{V}_{\mathscr{S}} \\
\stackrel{\mu^{t} \otimes \phi \otimes 1}{\longrightarrow} S_{2} \mathscr{V}_{\mathscr{S}} \otimes \mathscr{V}_{\mathscr{S}} \stackrel{\text { mult }}{\longrightarrow} S_{3} \mathscr{V}_{\mathscr{S}}
\end{aligned}
$$

is the zero map. This can be verified fiber by fiber.

Now we turn to the normal bundle of $\mathscr{I}$ in $\mathscr{X}$. Recall that the map $f: \mathscr{H} \rightarrow \mathscr{X}$ forgets the plane cubic part of a curve $C$ in $\mathscr{Y}$. The fiber of $f$ over $f(\mid C]) \in \mathscr{I}$ consists therefore of all cubics in the plane given by $f([C])$ passing doubly through the distinguished point given by $f([C])$.

Let $p: \mathbf{P}(\mathscr{2}) \rightarrow \mathscr{I}$ be the structure map and let $v: \mathscr{Q}_{\mathbf{P}(2)} \rightarrow \mathcal{O}_{\mathbf{P}(2)}(\tau)$ be the tautological quotient linebundle. Then the bundle of "cubic" forms" on $\mathbf{P}(2)$ is $p_{*} \mathcal{O}_{\mathbf{P}(2)}(3 \tau)=S_{3} 2$. The cubics we are interested in are those vanishing to the second order along $\Sigma$. Thus they are sections of the kernel of the natural restriction map $S_{3} \mathscr{2} \rightarrow p_{*} \mathcal{O}_{\Sigma_{2}}(3 \tau)$, where $\Sigma_{2}$ is the full first-order neighbourhood of $\Sigma$ in $\mathbf{P}(2)$.

LEMMA 4.2. There is an exact sequence

$$
0 \rightarrow \mathscr{D}(2 \xi) \rightarrow p_{*} \mathcal{O}_{\Sigma_{2}}(3 \tau) \rightarrow \mathcal{O}_{\mathscr{I}}(3 \xi) \rightarrow 0 .
$$

Proof. By definition, $\Sigma \subseteq \mathbf{P}(\mathscr{2})$ is the locus where the two quotients $v: \mathscr{Q}_{\mathbf{P}(2)} \rightarrow \mathcal{O}_{\mathbf{P}(2)}(\tau)$ and $\lambda: \mathscr{Q}_{\mathbf{P}(2)} \rightarrow \mathcal{O}_{\mathbf{P}(2)}(\xi)$ coincide, in other words the zero scheme of the composed map $\mathscr{D}_{\mathbf{P}(2)} \stackrel{\phi^{\prime}}{\longrightarrow} \mathscr{Q}_{\mathbf{P}(2)} \stackrel{\mathcal{}}{\rightarrow} \mathcal{O}_{\mathbf{P}(2)}(\tau)$. It follows that the conormal bundle of $\Sigma$ in $\mathbf{P}(\mathscr{2})$ is $\mathscr{D}_{\Sigma}(-\tau)$. Thus there is an exact sequence:

$$
0 \rightarrow \mathscr{D}_{\Sigma}(-\tau) \rightarrow \mathcal{O}_{\Sigma_{2}} \rightarrow \mathcal{O}_{\Sigma} \rightarrow 0 .
$$

Twisting by $3 \tau$ and applying $p_{*}$, we get the exact sequence on $\mathscr{I}$ :

$$
0 \rightarrow p_{*} \mathscr{D}_{\Sigma}(2 \tau) \rightarrow p_{*} \mathcal{O}_{\Sigma_{2}}(3 \tau) \rightarrow p_{*} \mathcal{O}_{\Sigma}(3 \tau) \rightarrow 0 .
$$

Finally, using that $\tau=\xi$ on $\Sigma$, we are done. 
PROPOSITION 4.3. There is an exact sequence

$$
0 \rightarrow N_{\mathscr{I} / \mathscr{X}} \rightarrow \operatorname{det}(\mathscr{B})^{-1} \otimes S_{3} \mathscr{Q} \rightarrow \operatorname{det}(\mathscr{B})^{-1} \otimes p_{*} \mathcal{O}_{\Sigma_{2}}(3 \tau) \rightarrow 0 .
$$

Proof. Consider the diagram:

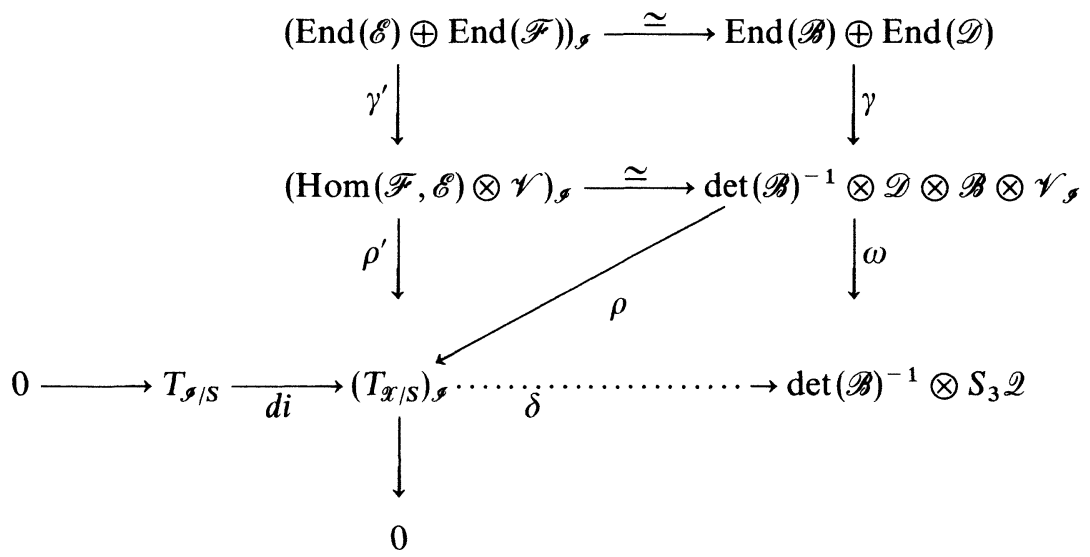

The horizontal isomorphisms are those induced by lemma 4.1. The vertical sequence is the restriction to $\mathscr{I}$ of an exact sequence

$$
0 \rightarrow \mathcal{O}_{\mathscr{X}} \rightarrow \operatorname{End}(\mathscr{E}) \oplus \operatorname{End}(\mathscr{F}) \stackrel{\gamma^{\prime}}{\longrightarrow} \operatorname{Hom}(\mathscr{F}, \mathscr{E} \otimes \mathscr{V}) \rightarrow T_{\mathscr{X} / S} \rightarrow 0
$$

where for a local section $(g, h)$ of $\operatorname{End}(\mathscr{E}) \oplus \operatorname{End}(\mathscr{F})$, we have $\gamma^{\prime}(g, h)=$ $\left(g \otimes 1_{\mathscr{V}}\right) \circ \mathscr{A}-\mathscr{A} \circ h$. The map $\omega$ is given as the identity on $\operatorname{det}(\mathscr{B})^{-1}$ times the composition

$$
\mathscr{D} \otimes \mathscr{B} \otimes \mathscr{V}_{\mathscr{g}} \stackrel{\phi^{\prime} \otimes \theta \otimes \psi^{\prime}}{\longrightarrow} \mathscr{Q} \otimes \mathscr{Q} \otimes \mathscr{2} \stackrel{\text { mult }}{\longrightarrow} S_{3} \mathscr{2} .
$$

Translating $\gamma^{\prime}$ via lemma 4.1, the map $\gamma$ takes the following value on a local section $(g, h)$ of $\operatorname{End}(\mathscr{B}) \oplus \operatorname{End}(\mathscr{D})$ :

$$
\begin{gathered}
\gamma(g, h)\left(\beta_{0} \wedge \beta_{1} \wedge \beta_{2}\right)= \\
\sum_{\sigma \in S_{3}} \operatorname{sign}(\sigma)\left(\psi \beta_{\sigma(0)} \otimes g \beta_{\sigma(1)} \otimes \phi \beta_{\sigma(2)}-h \psi \beta_{\sigma(0)} \otimes \beta_{\sigma(1)} \otimes \phi \beta_{\sigma(2)}\right) .
\end{gathered}
$$

From this formula we may verify that $\omega \circ \gamma=0$. Indeed, for a local section $(g, h)$ of $\operatorname{End}(\mathscr{B}) \oplus \operatorname{End}(\mathscr{D})$, we have

$$
\begin{gathered}
\omega \gamma(g, h)\left(\beta_{0} \wedge \beta_{1} \wedge \beta_{2}\right)= \\
\sum_{\sigma \in S_{3}} \operatorname{sign}(\sigma)\left(\theta \beta_{\sigma(0)} \cdot \theta g \beta_{\sigma(1)} \cdot \theta \beta_{\sigma(2)}-\phi^{\prime} h \psi \beta_{\sigma(0)} \cdot \theta \beta_{\sigma(1)} \cdot \theta \beta_{\sigma(2)}\right),
\end{gathered}
$$

in $S_{3}$ 2. The first part gives zero by the symmetry of the first and third factors, and the second part gives zero by the symmetry of the last two factors. 
It follows that there exists a map $\delta$ as above making the diagram commutative. Consider $\operatorname{Im}(\omega)$. From the description of $\omega$, it is clear that in a fiber over $x \in \mathscr{I}$, we have that $\operatorname{Im}(\omega) \otimes k(x)$ is the space of cubic forms in $S_{3} \mathscr{D}(x)$ which vanish to the second order in the distinguished point, since the ideal of this point in the plane $\mathbf{P}(\mathscr{2}(x))$ is generated by the image of $\phi^{\prime}(x): \mathscr{D}(x) \rightarrow \mathscr{2}(x)$. It follows that $\operatorname{Im}(\omega)$ fits into an exact sequence like the one in the proposition. It remains to prove that $N_{\mathscr{g} / \mathscr{X}}=\operatorname{Im}(\omega)$.

The map $d i$ is the differential of the inclusion $i: \mathscr{I} \rightarrow \mathscr{X}$, hence its cokernel is $N_{\mathscr{g} / \mathscr{P}}$. We proceed now to show that $\delta \circ d i=0$. Once we have done that, $\delta$ will have to factor through some map $N_{\mathscr{F} / \mathscr{X}} \rightarrow \operatorname{Im}(\omega)$, which will be surjective since $\operatorname{Im}(\delta)=\operatorname{Im}(\omega)$. Both $N_{\mathscr{I} / \mathscr{C}}$ and $\operatorname{Im}(\omega)$ are rank-7 bundles, so it is even an isomorphism.

To see that $\delta \circ d i=0$, pick a closed point $x \in \mathscr{I}$ and a tangent vector $t \in T_{\mathscr{I} / S}(x)$. This may be thought of as a morphism $\tilde{t}$ : Spec $k[\varepsilon] / \varepsilon^{2} \rightarrow \mathscr{I}$ centered in $x$. Consider the pullback via $\tilde{t}^{*}$ of the diagram (4-1). Each of $\tilde{t}^{*} \mathscr{V}_{\mathscr{I}}, \tilde{t}^{*} \mathscr{Q}, \tilde{t}^{*} \mathscr{B}$ and $\tilde{t}^{*} \mathscr{D}$ are free $k[\varepsilon]$-modules. So we may write $\tilde{t}^{*} \phi=\phi_{0}+\varepsilon \phi_{1}$, and similarly $\tilde{t}^{*} \psi=\psi_{0}+\varepsilon \psi_{1}$. Now the image $d i(t) \in T_{\mathscr{x}}(i(x))$ is represented by the composition

$$
\operatorname{Spec} k[\varepsilon] / \varepsilon^{2} \stackrel{\tilde{t}}{\rightarrow} \mathscr{I} \stackrel{i}{\rightarrow} \mathscr{X} .
$$

Using lemma 4.1, we may describe this as $\operatorname{di}(t)=\rho\left(t^{\prime}\right)$, where $t^{\prime} \in \operatorname{det}(\mathscr{B})^{-1} \otimes$ $\mathscr{D} \otimes \mathscr{B} \otimes \mathscr{V}_{\mathscr{I}}$ is given by the $\varepsilon$-component of the formula

$$
\beta_{0} \wedge \beta_{1} \wedge \beta_{2} \mapsto \sum_{\sigma \in S_{3}} \operatorname{sign}(\sigma)\left(\psi_{0}+\varepsilon \psi_{1}\right) \beta_{\sigma(0)} \otimes \beta_{\sigma(1)} \otimes\left(\phi_{0}+\varepsilon \phi_{1}\right) \beta_{\sigma(2)}
$$

Expanding this we get the following expression for $t^{\prime}$ :

$$
\beta_{0} \wedge \beta_{1} \wedge \beta_{2} \mapsto \sum_{\sigma \in S_{3}} \operatorname{sign}(\sigma)\left(\psi_{1} \beta_{\sigma(0)} \otimes \beta_{\sigma(1)} \otimes \phi_{0} \beta_{\sigma(2)}+\psi_{0} \beta_{\sigma(0)} \otimes \beta_{\sigma(1)} \otimes \phi_{1} \beta_{\sigma(2)}\right)
$$

Therefore $\delta(\operatorname{di}(t))=\omega\left(t^{\prime}\right)$ is given by

$$
\begin{aligned}
\beta_{0} \wedge \beta_{1} \wedge \beta_{2} \mapsto & \sum_{\sigma \in S_{3}} \operatorname{sign}(\sigma) \phi_{0}^{\prime} \psi_{1} \beta_{\sigma(0)} \cdot \theta \beta_{\sigma(1)} \cdot \theta \beta_{\sigma(2)}+ \\
& \sum_{\sigma \in S_{3}} \operatorname{sign}(\sigma) \theta \beta_{\sigma(0)} \cdot \theta \beta_{\sigma(1)} \cdot \psi_{0} \phi_{1}^{\prime} \beta_{\sigma(2)} .
\end{aligned}
$$

The first of these sums vanish because of the symmetry in the last two factors, the second sum vanishes because of the symmetry in the first two factors. We conclude that $\delta \circ d i=0$, which completes the proof.

\section{Blowing up the degenerate nets.}

The aim of this section is twofold. We want to prove that $\mathscr{H}$ is isomorphic to the blow up of $\mathscr{X}$ along $\mathscr{I}$, and we want to give an explicit relation between the 
universal curve $\tilde{\mathscr{C}} \subseteq \mathscr{P}_{\mathscr{H}}$ and the universal degeneration locus $\mathscr{Z} \subseteq \mathscr{P}_{\mathscr{P}}$. Since the invariants of $\mathscr{Z}$ are easy to express in terms of the universal bundles $\mathscr{E}$ and $\mathscr{F}$ on $\mathscr{X}$, this enables us to compute the Chern classes of $\pi_{\mathscr{H} *} \mathcal{O}_{\tilde{\mathscr{E}}}(5)$.

Let $\Phi: \tilde{\mathscr{X}} \rightarrow X$ be the blow up of $\mathscr{I}$ and let $\tilde{\mathscr{Y}}$ denote the exceptional divisor. To produce a map $\tilde{X} \rightarrow \mathscr{H}$ over $\mathscr{X}$, we have to construct a subvariety $\tilde{\mathscr{C}}^{\prime} \subseteq \mathscr{P}_{\tilde{\mathscr{X}}}$ which is flat over $\tilde{\mathscr{X}}$ and which coincides with $\mathscr{Z}$ off the exceptional divisor. There is just one choice available, namely let $\tilde{\mathscr{C}}^{\prime}$ be the closure of $\left(\pi^{*} \Phi\right)^{-1}\left(\left.\mathscr{Z}\right|_{\mathscr{x}-g}\right)$ in $\mathscr{P}_{\tilde{x}}$. We shall show below that this is indeed flat, but first we need some more notation.

First, let $g: \tilde{Y} \rightarrow I$ be the restriction of $\Phi$. From general theory, we know that $\tilde{\mathscr{Y}}=\mathbf{P}\left(N_{\mathscr{F} / \mathscr{X}}^{\vee}\right)$ with structure morphism $g$. Let $\left(N_{\mathscr{I} / \mathscr{X}}^{\vee}\right) \tilde{\mathscr{y}} \rightarrow \mathcal{O}_{\tilde{y}}(\eta)$ be the tautological quotient.

Let $W$ be the pullback via $g$ of the universal plane $\mathbf{P}(\mathscr{Q}) \subseteq \mathscr{P}_{\mathscr{S}}$, i.e., $W=\mathbf{P}\left(g^{*} \mathscr{Q}\right) \subseteq \mathscr{P}_{\tilde{y}}$. Let $\mathscr{Q}_{W} \rightarrow \mathcal{O}_{W}(\tau)$ be the corresponding tautological quotient.

Putting these maps together, we obtain maps on $W$, where the middle map is induced from prop. 4.3:

$$
\mathcal{O}_{W}(-\eta) \otimes \operatorname{det}(\mathscr{B}) \rightarrow\left(N_{\mathscr{S} / \mathscr{x}}\right)_{W} \otimes \operatorname{det}(\mathscr{B}) \rightarrow S_{3} \mathscr{2}_{W} \rightarrow \mathcal{O}_{W}(3 \tau) .
$$

The composition of these gives a global section of $\mathcal{O}_{W}(\eta-\beta+3 \tau)$, where we write $\beta$ for the divisor class of $\operatorname{det}(\mathscr{B})$. The locus where this section vanishes will be denoted by $\Pi \subseteq W$. Its fiber over a point in $\tilde{\mathscr{Y}}$ is exactly the singular plane cubic corresponding to that point. Hence we may refer to $\Pi$ as the "universal singular plane cubic".

Proposition 5.1. (i) $\mathscr{C}^{\prime}$ is flat over $\tilde{\mathscr{X}}$.

(ii) $\mathscr{Z}_{\tilde{x}}=W \cup \mathscr{C}^{\prime}$.

(iii) $W \cap \tilde{\mathscr{C}}^{\prime}=\Pi$.

Proof. We shall locally on $\mathscr{X}$ make an explicit construction of the blowing up where we can compute the ideal of $\mathscr{Z}_{\tilde{x}}$. Let $u \in \mathscr{I}$ be a closed point and let $U_{0} \subseteq \mathscr{I}$ be an open neighbourhood of $u$ which is small enough that $\mathscr{V}_{U_{0}}$ has four linearly independent sections $x_{0}, x_{1}, x_{2}, x_{3}$ such that everywhere on $U_{0}, x_{0}=0$ defines the distinguished plane and $x_{2}=x_{1}=x_{0}=0$ defines the distinguished point. In other words, $x_{0}$ generates the subbundle $\mathcal{O}_{U_{0}}\left(-\xi^{\vee}\right)$ of $\mathscr{V}_{U_{0}}$ and $x_{2}, x_{1}, x_{0}$ generate the subbundle $\mathscr{B}_{U_{0}}$.

Let $\mathbf{A}^{7}=\operatorname{Spec} k\left[a_{1}, a_{3}, b_{1}, b_{2}, b_{3}, c_{2}, c_{3}\right]$, where the variables are algebraically independent. On $\mathbf{A}^{7} \times U_{0}$ there are sections

$$
\begin{aligned}
& a=a_{1} x_{1}+a_{3} x_{3} \\
& b=b_{1} x_{1}+b_{2} x_{2}+b_{3} x_{3} \\
& c=c_{2} x_{2}+c_{3} x_{3}
\end{aligned}
$$

of $\mathscr{V}_{\mathbf{A}^{7} \times U_{0}}$, and we may consider the matrix 


$$
\left(\begin{array}{cc}
c & b-x_{0} \\
b+x_{0} & a \\
-x_{1} & x_{2}
\end{array}\right)
$$

The minors of this matrix are linearly independent in some open affine neighborhood $U=\operatorname{Spec}(R) \subseteq \mathbf{A}^{7} \times U_{0}$ of $\{0\} \times U_{0}$, where $0 \in \mathbf{A}^{7}$ denotes the origin. Hence there is a morphism $t: U \rightarrow \mathscr{X}$ which restricts to the inclusion of $U_{0} \subseteq \mathscr{I}$ in $\mathscr{X}$ on the subvariety $\{0\} \times U_{0}$.

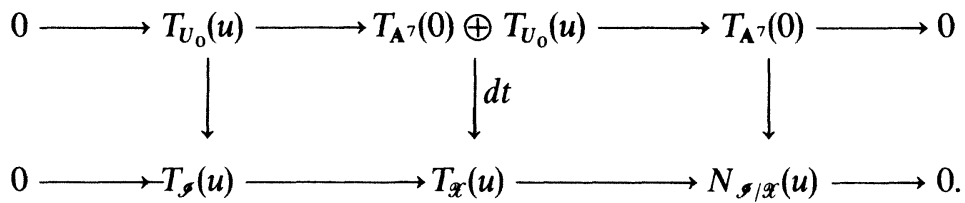

Here the left vertical map is the derivative of an open inclusion, hence an isomorphism. The right vertical map is an isomorphism by construction, prop 4.3, and remark (ii) after lemma 2.1. It follows that $d t$ is an isomorphism, hence $t$ is étale at the point $(0, u)$.

Now let $\tilde{U}$ be some open affine subset of the blow up of $U$ along $\{0\} \times U_{0}$. There is then a diagram

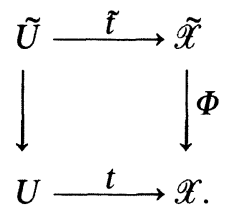

Since $t$ is étale at $(0, u)$, it follows that $t$ is étale at any point lying over $(0, u)$.

Let $R^{\prime} \supseteq R$ be an overring in which the ideal $J=\left(a_{1}, a_{3}, b_{1}, b_{2}, b_{3}, c_{2}, c_{3}\right)$ is invertible, say $J R^{\prime}=(y) R^{\prime}$ for some non-zero-divisor $y \in R^{\prime}$. For example, $R^{\prime}=\Gamma\left(\tilde{U}, \mathcal{O}_{\tilde{U}}\right)$ is of this type. Put $U^{\prime}=\operatorname{Spec}\left(R^{\prime}\right)$. Define $a_{i}^{\prime}, b_{i}^{\prime}, c_{i}^{\prime} \in R^{\prime}$ by $a_{i}=y a_{i}^{\prime}$, $b_{i}=y b_{i}^{\prime}$, and $c_{i}=y c_{i}^{\prime}$. Also, let $a^{\prime}, b^{\prime}$, and $c^{\prime}$ be similarly defined sections of $\mathscr{V}_{U^{\prime}}$, thus by $a=y a^{\prime}, b=y b^{\prime}$, and $c=y c^{\prime}$. On $\mathscr{P}_{U^{\prime}}$ we may write down the following complex $K^{\bullet}$ :

$$
\begin{aligned}
0 \rightarrow \mathcal{O}_{\mathscr{P}_{U}}(-4) & \stackrel{\phi}{\rightarrow} 3 \mathcal{O}_{\mathscr{P}_{U}}(-3) \oplus \mathcal{O}_{\mathscr{P}_{U}}(-4) \\
& \stackrel{\Psi}{\rightarrow} 3 \mathcal{O}_{\mathscr{P}_{U}}(-2) \oplus \mathcal{O}_{\mathscr{P}_{U}}(-3) \stackrel{\mu}{\rightarrow} \mathcal{O}_{\mathscr{P}_{U}},
\end{aligned}
$$

where

$$
\phi=\left(\begin{array}{c}
x_{2} \\
x_{1} \\
x_{0} \\
-y
\end{array}\right), \psi=\left(\begin{array}{cccc}
y c^{\prime} & y b^{\prime}-x_{0} & x_{1} & c^{\prime} x_{2}+b^{\prime} x_{1} \\
y b^{\prime}+x_{0} & y a^{\prime} & -x_{2} & b^{\prime} x_{2}+a^{\prime} x_{1} \\
-x_{1} & x_{2} & 0 & 0 \\
0 & 0 & -y & -x_{0}
\end{array}\right), \mu=\left(\delta_{1}, \delta_{2}, \delta_{3}, F\right),
$$


where $\delta_{i}$ is the $i$ th $2 \times 2$ minor of the upper left $3 \times 2$ submatrix of $\psi$ and $F=a^{\prime} x_{1}^{2}+2 b^{\prime} x_{1} x_{2}+c^{\prime} x_{2}^{2}$. It is straightforward to check that this is in fact a complex. Let $C^{\prime} \subseteq \mathscr{P}_{U^{\prime}}$ be defined by $\mathcal{O}_{\mathbf{C}^{\prime}}=\operatorname{Coker}(\mu)$.

Lemma 5.2. Assume that $F$ is not a zero-divisor in $R^{\prime} /(y) R^{\prime}\left[x_{1}, x_{2}, x_{3}\right]$. Then there is an open neighborhood $U^{\prime \prime}$ of the locus $V(y)$ in $U^{\prime}$ such that the complex $K_{\mathscr{U}^{\prime \prime}}{ }^{\prime \prime}$ is a resolution of $C_{U^{\prime \prime}}^{\prime}$. Furthermore, $C_{U^{\prime \prime}}^{\prime \prime}$ is flat over $U^{\prime \prime}$.

Proof. For any $x \in V(y)$ it follows from lemma 2.1 that $K^{\bullet} \otimes k(x)$ is a resolution. The same will then be true for all $x$ in a neighborhood $U^{\prime \prime}$ of $V(y)$ in $U^{\prime}$. But then the complex itself is a resolution of $\mathcal{O}_{C_{U \prime \prime}^{\prime}}$, which implies flatness as well.

Lemma 5.3. Assume that $F$ is not a zero-divisor in $R^{\prime} /(y) R^{\prime}\left[x_{1}, x_{2}, x_{3}\right]$. Then

(i) $\left(\delta_{1}, \delta_{2}, \delta_{3}, F\right) \cap\left(x_{0}, y\right)=\left(\delta_{1}, \delta_{2}, \delta_{3}\right)$.

(ii) $\left(\delta_{1}, \delta_{2}, \delta_{3}, F\right)+\left(x_{0}, y\right)=\left(x_{0}, y, F\right)$.

ProOF. First observe that each of the minors $\delta_{i}$ is contained in the ideal $\left(x_{0}, y\right)$. Hence $\left(\delta_{1}, \delta_{2}, \delta_{3}, F\right) \cap\left(x_{0}, y\right) \supseteq\left(\delta_{1}, \delta_{2}, \delta_{3}\right)$. To prove the opposite inclusion, remark that both $x_{0} F$ and $y F$ are elements of $\left(\delta_{1}, \delta_{2}, \delta_{3}\right)$, and by the assumption on $F$, it follows that $(F) \cap\left(x_{0}, y\right)=\left(F x_{0}, F y\right) \subseteq\left(\delta_{1}, \delta_{2}, \delta_{3}\right)$. This proves (i). (ii) follows easily from $\delta_{i} \in\left(x_{0}, y\right)$.

To complete the proof of prop. 5.1, observe that set-theoretically, the equality $\mathscr{Z}_{\tilde{x}}=W \cup \widetilde{\mathscr{C}}^{\prime}$ holds. An easy dimension count shows that both $W$ and $\tilde{\mathscr{C}}^{\prime}$ are of codimension two in $\mathscr{P}_{\tilde{x}}$. Since $\mathscr{Z}_{\tilde{x}}$ is determinantal, it has no imbedded components, hence (ii) holds.

Now let $u \in \tilde{\mathscr{Y}}$ be any point and choose an open affine neighborhood $U^{\prime}=\operatorname{Spec}\left(R^{\prime}\right)$ of $u$ in $\tilde{\mathscr{X}}$, small enough that the complex $K_{\dot{\mathscr{P}}_{U^{\prime}}}$ is a resolution. The ring $R^{\prime}$ being regular in this case, it is clear that in the notation above, $F$ is not a zero-divisor in $R^{\prime} /(y) R^{\prime}\left[x_{1}, x_{2}, x_{3}\right]$. Hence, shrinking $U^{\prime}$ if necessary, we may assume that the conclusions of lemmas 5.2 and 5.3 hold. Observe that $t^{-1}(W)$ is given by the ideal $\left(x_{0}, y\right)$ and that $\left(\pi^{*}(\Phi \circ \tilde{t})\right)^{-1}(\mathscr{Z})$ is given by $\left(\delta_{1}, \delta_{2}, \delta_{3}\right)$.

By lemma 5.3 it follows that $\tilde{t}^{-1}\left(\widetilde{\mathscr{C}}^{\prime}\right)$ is given by the ideal $\left(\delta_{1}, \delta_{2}, \delta_{3}, F\right)$. Hence, since $u \in \tilde{\mathscr{Y}}$ was arbitrary, the scheme $\tilde{t}^{-1}\left(\tilde{\mathscr{C}}^{\prime}\right)$ is flat over $\tilde{X}$ by lemma 5.2 . By lemma 5.3 (ii), it follows that $W \cap \widetilde{\mathscr{C}}^{\prime}$ equals $\Pi$ scheme-theoretically.

COROLlary 5.4. There is an isomorphism $\kappa: \tilde{\mathscr{X}} \rightarrow \mathscr{H}$ such that $f \circ \kappa=\Phi$. Under this isomorphism, $\mathscr{C}^{\prime}=\left(\pi^{*} \kappa\right)^{-1} \tilde{\mathscr{C}}$.

Proof. By prop. 5.1, it follows that there exists a morphism $\kappa: \tilde{X} \rightarrow \mathscr{H}$ such that $\mathscr{C}^{\prime}=\left(\pi^{*} \kappa\right)^{-1} \tilde{\mathscr{C}}$. By construction, we have $\mathrm{f} \circ \kappa=\Phi$. It follows from the classification of the curves in $\mathscr{Y}$ that $\kappa$ is bijective. Since $\mathscr{H}$ is nonsingular, we conclude by Zariski's main theorem. 
From now on we will identify $\tilde{\mathscr{X}}$ with $\mathscr{H}$ via $\kappa$; thus $f=\Phi$ and $\tilde{\mathscr{C}}^{\prime}=\tilde{\mathscr{C}}$. Write $\mathscr{Z}_{\mathscr{H}}=\left(\pi^{*} f\right)^{-1}(\mathscr{Z})$.

COROLlaRY 5.5. Under these identifications, we have

(i) $W \cup \tilde{\mathscr{C}}=\mathscr{Z}_{\mathscr{H}}$.

(ii) $W \cap \tilde{\mathscr{C}}=\Pi$.

(iii) There is an exact sequence

$$
0 \rightarrow \mathscr{I}_{\Pi / W} \rightarrow \mathcal{O}_{\mathscr{X}_{\mathscr{H}}} \rightarrow \mathcal{O}_{\tilde{\mathscr{C}}} \rightarrow 0 .
$$

ProOF. (i) and (ii) are just restatements of prop. 5.1 (ii) and (iii). The exact sequence follows easily from the exact sequence $0 \rightarrow \mathcal{O}_{\mathscr{x}_{\mathscr{H}}} \rightarrow \mathcal{O}_{\tilde{\mathscr{E}}} \oplus \mathcal{O}_{W} \rightarrow \mathcal{O}_{\Pi} \rightarrow 0$, a consequence of (i) and (ii).

A useful consequence of cor. 5.5 is that $\mathscr{Z}_{\mathscr{H}}$ has codimension two in $P_{\mathscr{H}}$, which implies that the complex (3-2) stays exact after we pull it back to $\mathscr{P}_{\mathscr{H}}$ by $\pi^{*} f$. It follows that

Proposition 5.6. For any $i \geqq 0$, in particular for $i=5$,

$$
f^{*} c\left(\pi_{\mathscr{X} *} \mathcal{O}_{\mathscr{Z}}(i)\right)=\mathrm{c}\left(\pi_{\mathscr{H} *} \mathcal{O}_{\mathscr{X}_{\mathscr{H}}}(i)\right) .
$$

\section{Identification of the Chern class.}

We turn to the detailed Chern class computations necessary to calculate the top Chern class $c_{16}\left(\pi_{\mathscr{X}} \mathcal{O}_{\tilde{\mathscr{C}}}(5)\right)$. As we shall prove in the next section, the cohomology ring (and the Chow ring) of $\mathscr{X}$ is generated by the Chern classes of $\mathscr{E}, \mathscr{F}$, and $\mathscr{V}$. Hence we seek to express $c\left(\pi_{\mathscr{X}_{*}} \mathcal{O}_{\tilde{\mathscr{C}}}(5)\right)$ in terms of these.

In order to avoid an explicit description of the Chow ring of $\mathscr{H}$ in terms of generators and relations, and also to keep computations within reasonable size, we split the expression for $\int_{\mathscr{H}} c\left(\pi_{\mathscr{X} *} \mathcal{O}_{\tilde{\mathscr{C}}}(5)\right)$ in two parts, one contribution called the "main term" coming from $\mathscr{X}$ and one, called "the correction term", coming from the exceptional divisor $\mathscr{Y}$.

Recall that we have the following diagram with cartesian squares:

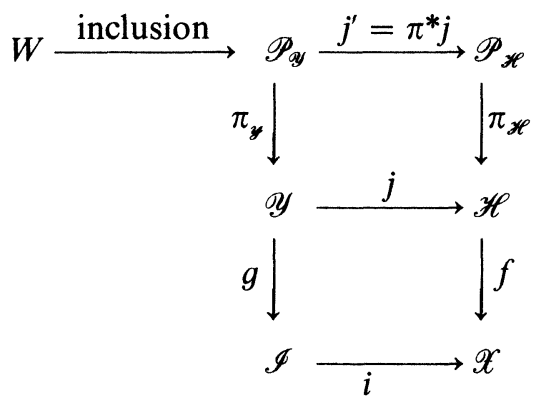


Consider the sequence

$$
0 \rightarrow \mathscr{I}_{\Pi / W} \rightarrow \mathcal{O}_{\mathscr{Z}_{\mathscr{H}}} \rightarrow \mathcal{O}_{\tilde{\mathscr{C}}} \rightarrow 0
$$

from corollary 5.5 (iii). Using that $\mathscr{I}_{\Pi / W}=\mathcal{O}_{W}(-\eta+\beta-3 \tau)$ by the definition of $\Pi$, this gives after twisting by $5 \tau$ :

$$
0 \rightarrow j_{*}^{\prime} \mathcal{O}_{W}(-\eta+\beta+2 \tau) \rightarrow \mathcal{O}_{\mathscr{X}_{*}}(5 \tau) \rightarrow \mathcal{O}_{\tilde{\mathscr{E}}}(5 \tau) \rightarrow 0 .
$$

Pushing this down to $\mathscr{H}$ via $\pi_{\mathscr{X}_{*}}$ yields

$$
0 \rightarrow \pi_{\mathscr{H} *} j_{*}^{\prime} \mathcal{O}_{W}(-\eta+\beta+2 \tau) \rightarrow \pi_{\mathscr{X}_{*}} \mathcal{O}_{\mathscr{X}_{*}}(5 \tau) \rightarrow \pi_{\mathscr{X} *} \mathcal{O}_{\tilde{\mathscr{G}}}(5 \tau) \rightarrow 0 .
$$

The first of these terms is simply

$$
\pi_{\mathscr{H} *} j_{*}^{\prime} \mathcal{O}_{W}(-\eta+\beta+2 \tau)=j_{*} \pi_{\mathscr{Y}_{*}} \mathcal{O}_{W}(-\eta+\beta+2 \tau)=j_{*} S_{2} \mathscr{Q}_{\mathscr{Y}}(-\eta+\beta)
$$

the second is

$$
\pi_{\mathscr{X}_{*}} \mathcal{O}_{\mathscr{P}_{*}}(5 \tau)=f^{*} \pi_{\mathscr{X} *} \mathcal{O}_{\mathscr{P}}(5) \text {. }
$$

Hence if we put $\mathscr{A}_{\mathscr{Q}}=S_{2} \mathscr{\mathscr { Q }}_{\mathscr{y}}(-\eta+\beta)$ and $\mathscr{A}_{\mathscr{X}}=\pi_{\mathscr{X}_{*}} \mathcal{O}_{\mathscr{X}}(5)$, we have the following exact sequence:

$$
0 \rightarrow j_{*} \mathscr{A}_{\mathscr{Y}} \rightarrow f^{*} \mathscr{A}_{x} \rightarrow \pi_{\mathscr{X} *} \mathcal{O}_{\tilde{\mathscr{E}}}(5 \tau) \rightarrow 0 .
$$

Here $\mathscr{A}_{y}$ is a locally free sheaf of rank 6 on $\mathscr{Y}$, and $\mathscr{A}_{x}$ is a coherent sheaf of rank 16 on $\mathscr{X}$, which furthermore fits into an exact sequence on $\mathscr{X}$ :

$$
0 \rightarrow \mathscr{F} \otimes S_{2} \mathscr{V}_{\mathscr{X}} \rightarrow \mathscr{E} \otimes S_{3} \mathscr{V}_{\mathscr{X}} \rightarrow S_{5} \mathscr{V}_{\mathscr{X}} \rightarrow \mathscr{A}_{\mathscr{X}} \rightarrow 0,
$$

obtained from (3-2) by twisting by 5 and applying $\pi_{\mathscr{X} *}$.

Lemma 6.1. Let $j: Y \rightarrow H$ be the inclusion of a divisor, $Y$ and $H$ both smooth varieties. Let $y=j^{*}[Y]=c_{1}\left(\mathcal{O}_{Y}(Y)\right)$. Suppose $\mathscr{A}$ is a locally free sheaf on $Y$. Then

$$
c\left(j_{*} \mathscr{A}\right)^{-1}=1-j_{*} \frac{c(\mathscr{A})-c(\mathscr{A}(-y))}{y c(\mathscr{A})} .
$$

Proof. First note that

$$
\left(1+j_{*} x\right)^{-1}=1-j * \frac{x}{1+y x}
$$

for all $x \in \mathscr{A}^{*}(Y)$. Indeed, since $j^{*} j_{*} x=y x$, we get by multiplying out:

$$
\begin{aligned}
& \left(1+j_{*} x\right)\left(1-j_{*} \frac{x}{1+y x}\right) \\
& \quad=1+j_{*} x-j_{*} \frac{x}{1+y x}-j_{*} x \cdot j_{*} \frac{x}{1+y x}
\end{aligned}
$$




$$
\begin{aligned}
& =1+j_{*} x-j_{*}\left(\left(j^{*} j_{*} x\right) \cdot \frac{x}{1+y x}\right) \text { by the projection formula } \\
& =1+j_{*} x-j_{*} \frac{x}{1+y x}-j_{*}\left(y x \cdot \frac{x}{1+y x}\right) \\
& =1+j_{*}\left(x-\frac{x}{1+y x}-y x \frac{x}{1+y x}\right) \\
& =1 .
\end{aligned}
$$

Next, Riemann-Roch without denominators [11, thm. 15.3] gives

$$
c\left(j_{*} \mathscr{A}\right)=1+j_{*} \frac{c(\mathscr{A})-c(\mathscr{A}(-y))}{y c(\mathscr{A}(-y))} .
$$

Now let $x=\frac{c(\mathscr{A})-c(\mathscr{A}(-y))}{y c(\mathscr{A}(-y))}$, and note that $1+y x=\frac{c(\mathscr{A})}{c(\mathscr{A}(-y))}$. Applying the above, we get

$$
\begin{aligned}
c\left(j_{*} \mathscr{A}\right)^{-1}=\left(1+j_{*} x\right)^{-1} & =1-j_{*}\left(\frac{x}{1+y x}\right) \\
& =1-j_{*} \frac{x c(\mathscr{A}(-y))}{c(\mathscr{A})} \\
& =1-j_{*} \frac{c(\mathscr{A})-c(\mathscr{A}(-y))}{y c(\mathscr{A})} .
\end{aligned}
$$

Returning to our situation, (6-1) gives in conjunction with lemma 6.1 the following equalities:

$$
\begin{aligned}
\int_{\mathscr{H}} c\left(\pi_{\mathscr{H}_{*}} \mathcal{O}_{\tilde{\mathscr{C}}}(5 \tau)\right) & =\int_{\mathscr{H}} c\left(f^{*} \mathscr{A}_{\mathscr{A}}\right) \cdot c\left(j_{*} \mathscr{A}_{\mathscr{Y}}\right)^{-1} \\
& =\int_{\mathscr{H}} c\left(f^{*} \mathscr{A}_{\mathscr{X}}\right) \cdot\left(1-j_{*} \frac{c\left(\mathscr{A}_{y}\right)-c\left(\mathscr{A}_{\mathscr{y}}(-y)\right)}{y c\left(\mathscr{A}_{\mathscr{y}}\right)}\right) \\
& =\int_{\mathscr{H}} f^{*} c\left(A_{\mathscr{X}}\right)-\int_{\mathscr{H}} f^{*} c\left(\mathscr{A}_{\mathscr{X}}\right) \cdot\left(j_{*} \frac{c\left(\mathscr{A}_{\mathscr{y}}\right)-c\left(\mathscr{A}_{\mathscr{y}}(-y)\right)}{y c\left(\mathscr{A}_{\mathscr{y}}\right)}\right) \\
& =\int_{\mathscr{X}} c\left(\mathscr{A}_{\mathscr{X}}\right)-\int_{\mathscr{y}} j^{*} f^{*} c\left(\mathscr{A}_{\mathscr{X}}\right) \cdot \frac{c\left(\mathscr{A}_{\mathscr{y}}\right)-c\left(\mathscr{A}_{y}(-y)\right)}{y c\left(\mathscr{A}_{y}\right)} \\
& =\int_{\mathscr{X}} c\left(\mathscr{A}_{\mathscr{X}}\right)-\int_{\mathscr{y}} c\left(g^{*} i^{*} \mathscr{A}_{\mathscr{X}}\right) \cdot \frac{c\left(\mathscr{A}_{\mathscr{y}}\right)-c\left(\mathscr{A}_{y}(-y)\right)}{y c\left(\mathscr{A}_{\mathscr{y}}\right)}
\end{aligned}
$$




$$
=\int_{\mathscr{X}} c\left(\mathscr{A}_{\mathscr{X}}\right)-\int_{\mathscr{I}} c\left(i^{*} \mathscr{A}_{X}\right) \cdot g_{*}\left(\frac{c\left(\mathscr{A}_{Y}\right)-c\left(\mathscr{A}_{Y}(-y)\right)}{y c\left(\mathscr{A}_{\mathscr{Y}}\right)}\right)
$$

We have proved

Proposition 6.2.

$$
\int_{\mathscr{H}} c\left(\pi_{\mathscr{X}_{*}} \mathcal{O}_{\tilde{\mathscr{E}}}(5 \tau)\right)=\int_{\mathscr{X}} c\left(\mathscr{A}_{\mathscr{X}}\right)+\int_{\mathscr{I}} c\left(i^{*} \mathscr{A}_{\mathscr{X}}\right) \cdot g_{*}\left(\frac{c\left(\mathscr{A}_{y}(-y)\right)-c\left(\mathscr{A}_{\mathscr{y}}\right)}{y c\left(\mathscr{A}_{y}\right)}\right)
$$

\section{Intersection rings.}

In this section we compute the Chow rings $A^{*}(X)$ and $A^{*}(\mathscr{X})$ by giving ring generators and relations. The approach given here is somewhat different from [9], where only he absolute case is treated. The advantage is that the present approach is better suited for generalizing to the relative case, e.g. the case of twisted cubic curves in $\mathbf{P}^{\mathbf{4}}$. It is also more oriented towards actually computing (on a computer) in the Chow ring.

We do the absolute case first. Recall the universal bundles $\mathscr{E}_{0}$ and $\mathscr{F}_{0}$ on $X$. Denote their Chern classes by $\gamma_{i}=c_{i}\left(\mathscr{E}_{0}\right)$ and $\delta_{i}=c_{i}\left(\mathscr{F}_{0}\right)$.

Proposition 7.1. $A^{*}(X)$ is generated as a Z-algebra by $\gamma_{1}, \gamma_{2}, \gamma_{3}, \delta_{1}$, and $\delta_{2}$.

REMARK. In fact, $\delta_{1}=\gamma_{1}$, so we may even omit $\delta_{1}$ from this list whenever convenient.

Proof. This can be found in [10] and goes as follows: Let

$$
\gamma: P=\operatorname{Isom}\left(\mathscr{E}_{0}, E\right) \times_{X} \operatorname{Isom}\left(\mathscr{F}_{0}, F\right) \rightarrow X
$$

be the principal $\mathrm{GL}(E) \times \mathrm{GL}(F)$-bundle associated to $\mathscr{E}_{0}$ and $\mathscr{F}_{0}$. Grothendieck proved [12] that $\gamma^{*}: A^{*}(X) \rightarrow A^{*}(P)$ is surjective with kernel generated by the $c_{i}\left(\mathscr{E}_{0}\right)$ and $c_{i}\left(\mathscr{F}_{0}\right),(i>0)$. On the other hand, $\gamma$ factors through the quotient map $W^{s} \rightarrow X$, so $\gamma^{*}$ factors through $A^{*}\left(W^{s}\right)=\mathrm{Z}$. The conclusion follows.

To study the relations, we shall exploit the Chern roots of the universal bundles on $X$. Let $\mathbf{F}\left(\mathscr{E}_{0}\right) \rightarrow X$ be the full flag bundle associated to $\mathscr{E}_{0}$, and let $\mathbf{F}_{0}=\mathbf{F}\left(\mathscr{E}_{0}\right) \times_{X} \mathbf{P}\left(\mathscr{F}_{0}\right)$ with structure morphism $\rho: \mathbf{F}_{0} \rightarrow X$. Let

$$
\rho^{*} \mathscr{E}_{0}=\mathscr{E}^{3} \rightarrow \mathscr{E}^{2} \rightarrow \mathscr{E}^{1} \rightarrow \mathscr{E}^{0}=0 \quad \text { and } \quad \rho^{*} \mathscr{F}_{0}=\mathscr{F}^{2} \rightarrow \mathscr{F}^{1} \rightarrow \mathscr{F}^{0}=0
$$

be (the pullbacks to $\mathbf{F}_{0}$ of) the universal flags. Hence the $\mathscr{E}^{i}$ and $\mathscr{F}^{i}$ are locally free of rank $i$. Also, let $\varepsilon_{i}=c_{1}\left(\mathscr{E}^{i}\right)-c_{1}\left(\mathscr{E}^{i-1}\right)$ for $i=1,2,3$ and $\phi_{i}=c_{1}\left(\mathscr{F}^{i}\right)-$ $c_{1}\left(\mathscr{F}^{i-1}\right)$ for $i=1$, 2. Then $\rho^{*}$ makes $A^{*}\left(\mathbf{F}_{0}\right)$ a free $A^{*}(X)$-module with basis

$$
\overline{\mathscr{B}}=\left\{\varepsilon_{1}^{i} \varepsilon_{2}^{j} \phi_{1}^{k} \mid 0 \leqq i \leqq 2,0 \leqq j, k \leqq 1\right\} .
$$


Note that $\gamma_{i}$ and $\delta_{j}$ are the elementary symmetric functions in the $\varepsilon_{i}$ and the $\phi_{j}$, respectively.

We want to present $A^{*}(X)$ as the quotient of some polynomial algebra. Let $R$ be the graded polynomial ring

$$
R=\mathrm{Z}\left[e_{1}, e_{2}, e_{3}, f_{1}, f_{2}\right]
$$

in the degree 1 variables $e_{i}$ and $f_{i}$. The group $W=S_{3} \times S_{2}$, where $S_{n}$ in the symmetric group on $n$ letters, acts linearly by permuting the variables in the natural way: If $w=(\sigma, \tau) \in W$, then $w \cdot \varepsilon_{i}=\varepsilon_{\sigma(i)}$ and $w \cdot f_{j}=e_{\tau(j)}$. Let $c_{i}$ (resp. $d_{i}$ ) be the $i$-th elementary symmetric polynomial in the $e_{i}$ (resp. $f_{i}$ ). Then the invariant ring $R^{W}$ is again a polynomial ring:

$$
R^{W}=\mathrm{Z}\left[c_{1}, c_{2} \cdot c_{3}, d_{1}, d_{2}\right] .
$$

Define surjective ring homomorphisms $\mu: R \rightarrow A^{*}\left(\mathbf{F}_{0}\right)$ and $\lambda: R^{W} \rightarrow A^{*}(X)$ by letting $\mu\left(e_{i}\right)=\varepsilon_{i}, \mu\left(f_{i}\right)=\phi_{i}, \lambda\left(c_{i}\right)=\gamma_{i}$, and $\lambda\left(d_{i}\right)=\delta_{i}$. Then the following diagram commutes, where $i: R^{W} \rightarrow R$ denotes the inclusion map:

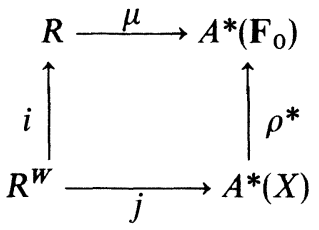

Furthermore, $R$ is a free $R^{W}$-module with basis

$$
\mathscr{B}=\left\{e_{1}^{i} e_{2}^{j} f_{1}^{k} \mid 0 \leqq i \leqq 2,0 \leqq j, k \leqq 1\right\},
$$

and the map $R \otimes_{R^{w}} A^{*}(X) \rightarrow A^{*}\left(\mathbf{F}_{0}\right)$ is an isomorphism.

Next we introduce the concept of "coordinate ideal": Let $\left\{b_{1}, \ldots, b_{12}\right\}$ be an $R^{W}$-basis for $R$. For any $r \in R$, let $c(r) \subseteq R^{W}$ be the ideal generated by the coordinates of $r \in R$ with respect to this basis. Precisely, if $r=\sum_{i=1}^{12} s_{i} b_{i}$ with $s_{i} \in R^{W}$, then $e(r)=\sum_{i} s_{i} R^{W}$ is the ideal generated by $s_{1}, \ldots, s_{12}$. Clearly $c(r)$ is independent of the choice of the basis $\left\{b_{i}\right\}$. If $J \subseteq R$ is an ideal, let $c(J) \subseteq R^{W}$ be the ideal generated by $\{c(r) \mid r \in J\}$.

We want to point out that with a good choice of the $\left\{b_{i}\right\}$, e.g., the one given by (7-2), the coordinates of a given element of $R$ can be effectively computed using elimination, for example by using a suitable Gröbner basis for the relations between the $e_{i}, f_{j}, c_{i}$, and $d_{j}$. See $[2,1,4]$.

LeMma 7.2. $\operatorname{Ker}(\lambda)=c(\operatorname{Ker}(\mu))$.

Proof. That $\operatorname{Ker}(\lambda) \subseteq c(\operatorname{Ker}(\mu))$ is straightforward. For the opposite inclusion, suppose $r=\sum_{i=1}^{12} s_{i} b_{i} \in \operatorname{Ker}(\mu)$, where $\left\{b_{1}, \ldots, b_{12}\right\}$ is a basis of $R$ over $R^{w}$, 
and $s_{i} \in R^{W}$. Then $\left\{\mu\left(b_{1}\right), \ldots, \mu\left(b_{12}\right)\right\}$ is a basis for $A^{*}\left(\mathbf{F}_{0}\right)$ over $A^{*}(X)$, so $0=\mu(r)=\sum_{i=1}^{12} \lambda\left(s_{i}\right) \mu\left(b_{i}\right)$ implies that $s_{i} \in \operatorname{Ker}(\lambda)$ for all $i$.

We proceed now to describe three explicit elements of $\operatorname{Ker}(\mu)$. Their coordinate ideals will be effectively computable and contained in $\operatorname{Ker}(\lambda)$.

LEMMA 7.3. $\operatorname{Ker}(\mu)$ contains the following three elements:

(i) $r_{0}=e_{1}+e_{2}+e_{3}-f_{1}-f_{2}$,

(ii) $r_{1}=\left(e_{1}-f_{1}\right)^{4}\left(e_{1}-f_{2}\right)^{4}$,

(iii) $r_{2}=\left(e_{1}-f_{2}\right)^{4}\left(e_{2}-f_{2}\right)^{4}$.

Proof. (i) is clear. For (ii), consider the diagram

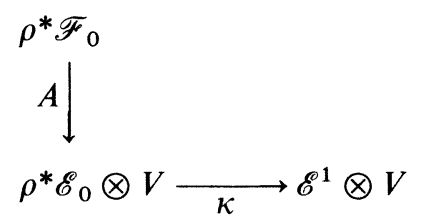

where $\kappa$ is induced from the natural quotient map $\rho^{*} \mathscr{E}_{0} \rightarrow \mathscr{E}^{1}$ and $A$ is the universal map. If the composed map $\kappa \circ A$ vanishes at some point $y \in \mathbf{F}_{0}$, then for a suitable choice of bases for $E$ and $F$, the map $A(x): F \rightarrow E \otimes V$, where $x=\rho(y)$, is represented by a matrix of the form

$$
\left(\begin{array}{ll}
0 & 0 \\
* & * \\
* & *
\end{array}\right)
$$

which contradicts the stability of $A(x)$. It follows that the global section

$$
\kappa \circ A \in H^{0}\left(\mathbf{F}_{0}, \rho^{*} \mathscr{F}_{0}^{\vee} \otimes \mathscr{E}^{1} \otimes V\right)
$$

has no zeroes, and hence the top Chern class $c_{8}\left(\rho^{*} \mathscr{F}_{0}^{\vee} \otimes \mathscr{E}^{1} \otimes V\right)$ vanishes. But this is exactly $\mu\left(r_{1}\right)$. The proof of (iii) is similar: Consider instead the diagram

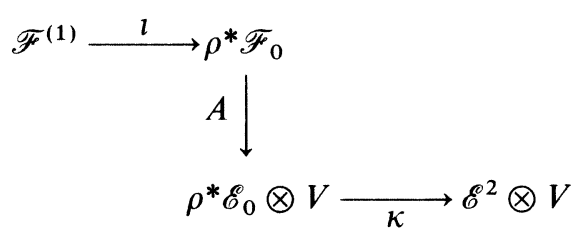

where $\mathscr{F}^{(1)}=\operatorname{Ker}\left(\rho^{*} \mathscr{F}_{0} \rightarrow \mathscr{F}^{1}\right)$ and $\imath$ the inclusion map, and $\kappa$ again is induced from the natural surjection map $\rho^{*} \mathscr{E}_{0} \rightarrow \mathscr{E}^{2}$. The composed map $\kappa \circ A \circ \imath$ has no zeroes, since stability prevents $A(x)$ from taking the form 


$$
\left(\begin{array}{ll}
0 & * \\
0 & * \\
* & *
\end{array}\right) .
$$

Hence the top Chern class of $\mathscr{F}^{(1)^{\vee}} \otimes \mathscr{E}^{2} \otimes V$ vanishes, and this class is precisely $\mu\left(r_{2}\right)$.

Put $\mathbf{b}=\sum_{i=0}^{2} c\left(r_{i}\right)=\left(c_{1}-d_{1}, c\left(\left(e_{1}-f_{1}\right)^{4}\left(e_{1}-f_{2}\right)^{4}\right), c\left(\left(e_{1}-f_{2}\right)^{4}\left(e_{2}-f_{2}\right)^{4}\right)\right.$ $\subseteq R^{W}$, where the $r_{i}$ are those of lemma 7.3. In view of lemma 7.2 we get

Table 1. Monomials and values for $A^{12}(X)$.

\begin{tabular}{|l|c|l|c|c|c|l|c|}
\hline$c_{1}^{12}$ & 119020 & $c_{1}^{4} c_{2}^{2} d_{2}^{2}$ & 1358 & $c_{1}^{5} c_{2}^{2} c_{3}$ & 1000 & $c_{1}^{2} c_{2}^{2} c_{3}^{2}$ & 62 \\
\hline$c_{1}^{10} c_{2}$ & 45748 & $c_{1}^{2} c_{2}^{3} d_{2}^{2}$ & 546 & $c_{1}^{3} c_{2}^{3} c_{3}$ & 410 & $c_{2}^{3} c_{3}^{2}$ & 28 \\
\hline$c_{1}^{8} c_{2}^{2}$ & 17772 & $c_{2}^{4} d_{2}^{2}$ & 225 & $c_{1} c_{2}^{4} c_{3}$ & 174 & $c_{1}^{4} d_{2} c_{3}^{2}$ & 102 \\
\hline$c_{1}^{6} c_{2}^{3}$ & 6996 & $c_{1}^{6} d_{2}^{3}$ & 2431 & $c_{1}^{7} d_{2} c_{3}$ & 1752 & $c_{1}^{2} c_{2} d_{2} c_{3}^{2}$ & 43 \\
\hline$c_{1}^{4} c_{2}^{4}$ & 2802 & $c_{1}^{4} c_{2} d_{2}^{3}$ & 955 & $c_{1}^{5} c_{2} d_{2} c_{3}$ & 698 & $c_{2}^{2} d_{2} c_{3}^{2}$ & 19 \\
\hline$c_{1}^{2} c_{2}^{5}$ & 1150 & $c_{1}^{2} c_{2}^{2} d_{2}^{3}$ & 381 & $c_{1}^{3} c_{2}^{2} d_{2} c_{3}$ & 284 & $c_{1}^{2} d_{2}^{2} c_{3}^{2}$ & 30 \\
\hline$c_{2}^{6}$ & 494 & $c_{2}^{3} d_{2}^{3}$ & 155 & $c_{1} c_{2}^{3} d_{2} c_{3}$ & 119 & $c_{2} d_{2}^{2} c_{3}^{2}$ & 13 \\
\hline$c_{2}^{10} d_{2}$ & 32302 & $c_{1}^{4} d_{2}^{4}$ & 676 & $c_{1}^{5} d_{2}^{2} c_{3}$ & 490 & $d_{2}^{3} c_{3}^{2}$ & 9 \\
\hline$c_{1}^{8} c_{2} d_{2}$ & 12492 & $c_{1}^{2} c_{2} d_{2}^{4}$ & 268 & $c_{1}^{3} c_{2} d_{2}^{2} c_{3}$ & 198 & $c_{1}^{3} c_{3}^{3}$ & 22 \\
\hline$c_{1}^{6} c_{2}^{2} d_{2}$ & 4890 & $c_{2}^{2} d_{2}^{4}$ & 108 & $c_{1} c_{2}^{2} d_{2}^{2} c_{3}$ & 82 & $c_{1} c_{2} c_{3}^{3}$ & 10 \\
\hline$c_{1}^{4} c_{2}^{3} d_{2}$ & 1944 & $c_{1}^{2} d_{2}^{5}$ & 190 & $c_{1}^{3} d_{2}^{3} c_{3}$ & 139 & $c_{1} d_{2} c_{3}^{3}$ & 7 \\
\hline$c_{1}^{2} c_{2}^{4} d_{2}$ & 789 & $c_{2} d_{2}^{5}$ & 76 & $c_{1} c_{2} d_{2}^{3} c_{3}$ & 57 & $c_{3}^{4}$ & 2 \\
\hline$c_{2}^{5} d_{2}$ & 331 & $d_{2}^{6}$ & 54 & $c_{1} d_{2}^{4} c_{3}$ & 40 & & \\
\hline$c_{1}^{8} d_{2}^{2}$ & 8826 & $c_{1}^{9} c_{3}$ & 6336 & $c_{1}^{6} c_{3}^{2}$ & 356 & & \\
\hline$c_{1}^{6} c_{2} d_{2}^{2}$ & 3438 & $c_{1}^{7} c_{2} c_{3}$ & 2496 & $c_{1}^{4} c_{2} c_{3}^{2}$ & 146 & & \\
\hline
\end{tabular}

Proposition 7.4. Let $\mathbf{b}$ and $\lambda$ be as above. Then $\mathbf{b} \subseteq \operatorname{Ker}(\lambda)$.

REMARK. In fact equality holds, at least if we allow rational coefficients; that is, $\left(R^{W} / \mathbf{b}\right) \otimes_{\mathbf{z}} \mathrm{Q} \simeq A_{0}^{*}(X)$. This is a special case of $[9$, thm. 4.4].

Proposition 7.5. For each monomial $M$ of degree 12 in $R^{W}$, we have $\int_{X} \lambda(M)=d(M)$, where the integers $d(M)$ are given in table 1 . 
Proof. First we compute that for each monomial $M$ as above, $2 M \equiv d(M) c_{3}^{4}$ $(\bmod \mathbf{b})$. This is a brute force Gröbner basis calculation. Next, it is clear that the $\int_{X} \lambda(M)$ are integers and that $\int_{X} \lambda(M)=\varepsilon d(M)$ for some constant $\varepsilon$. By proposition 7.1, the class of a point can be written as an integral linear combination of the $\lambda(M)$. It follows immediately that the integers $\int_{X} \lambda(M)$ are relatively prime. Since the $d(M)$ are also relatively prime integers, we get $\varepsilon= \pm 1$. It remains to show that at least one monomial is positive. For this, note that $-\gamma_{1}$ is the pullback of the Chern class of an ample linebundle under the inclusion morphism $X \rightarrow \operatorname{Grass}_{3}\left(S_{2} V\right)$. Hence $\gamma_{1}^{12}=\left(-\gamma_{1}\right)^{12}$ is positive, and we are done.

An alternative way to establish the class of a point is using the Gauss-Bonnet formula $\lambda_{\text {top }}(X)=\int_{X} c\left(T_{X}\right)$. We know [10] that the topological Euler characteristic of $X$ is 58, and we may compute (again using brute force) that the top Chern class of the tangent bundle $T_{X}$ is $c_{12}\left(T_{X}\right) \equiv 29 c_{3}^{4}(\bmod \mathbf{b})$. (The Chern class of $T_{X}$ is computed using (4-4).) This shows that the class of a point is $c_{3}^{4} / 2$.

We sketch how a few enumerative problems concerning twisted cubics can be solved using the intersection ring of the space $X$. Let $Z \subseteq P_{X}$ be the universal degeneration locus. By Porteous' formula, the class of $Z$ in $A^{2}\left(P_{X}\right)$ is

$$
[Z]=\left[c\left(\mathscr{E}_{0}(\tau)\right) / c\left(\mathscr{F}_{0}\right)\right]_{2}=3 \tau^{2}-\tau \gamma_{1}+\left(\gamma_{2}-\delta_{2}\right)
$$

where $\tau \in A^{1}\left(P_{X}\right)$ is the hyperplane class from $P=\mathbf{P}^{3}$.

Let $L$ be a line and $Q$ a point in $P$. Consider $X_{L}=\pi_{X}(Z \cap(L \times X))$ and $X_{Q}=\pi_{X}(Z \cap(Q \times X))$. Since $[L]=\tau^{2}$ and $[Q]=\tau^{3}$, we get

$$
\left[X_{L}\right]=\pi_{*}\left(\tau^{2}[Z]\right)=-\gamma_{1} \quad \text { and } \quad\left[X_{Q}\right]=\pi_{*}\left(\tau^{3}[Z]\right)=\gamma_{2}-\delta_{2} .
$$

Note that $X_{L}$ is the closure of the set of twisted cubics which intersect $L$, and $X_{Q}$ is the closure of the set of twisted cubics containing $Q$. Furthermore, let

$$
X_{B}=\left\{x \in X \mid l\left(Z_{x} \cap L\right) \geqq 2\right\}=\operatorname{Supp} R^{1} \pi_{X *}\left(\mathscr{I}_{Z} \otimes \mathcal{O}_{L \times X}\right)
$$

be the closure of the set of twisted cubics bisecant to $L$. From the sequence

$$
0 \rightarrow \pi_{X}^{*} \mathscr{F}_{0}(-3 \tau) \rightarrow \pi_{X}^{*} \mathscr{E}_{0}(-2 \tau) \rightarrow \mathscr{I}_{Z} \rightarrow 0
$$

we obtain, after tensorization with $\mathcal{O}_{L}$ and taking direct images, a presentation

$$
2 \mathscr{F}_{0} \rightarrow \mathscr{E}_{0} \rightarrow R^{1} \pi_{X *}\left(\mathscr{I}_{Z} \otimes \mathcal{O}_{L \times X}\right) \rightarrow 0 .
$$

Hence Porteous' formula gives

$$
\left[X_{B}\right]=\left[c\left(\mathscr{F}_{0}^{\vee}\right)^{2} / c\left(\mathscr{E}_{0}^{\vee}\right)\right]_{2}=2 \delta_{2}-\gamma_{2} .
$$

EXAMPLE 1. Let $p$ be an integer between 0 and 6 . The number of twisted cubics bisecant to $p$ given lines and passing through $6-p$ given points is $1,1,0,1,1,1$, 6 for $p=0,1,2,3,4,5,6$ respectively.. These numbers were found first by 
Cremona [7], who also gave explicit constructions for the twisted cubics in each case. We may compute these numbers as $\int_{X}\left(2 \delta_{2}-\gamma_{2}\right)^{p}\left(\gamma_{2}-\delta_{2}\right)^{6-p}$.

Example 2. In a given general $\mathbf{P}^{5}$ in the $\mathbf{P}^{9}=\mathbf{P}\left(S_{2} V^{\vee}\right)$ of all quadrics in $\mathbf{P}^{3}$ there are $\int_{X} \gamma_{3}^{4}=2$ determinantal nets of quadrics. This was proved by Reye $[18$, p. 79].

EXAMPLE 3. Let $L_{1}, \ldots, L_{12}$ be lines in general position. The intersection $X_{L_{i}} \cap \ldots \cap X_{L_{12}}$ contains the locus $I$ of degenerate nets. By [11, prop. 9.1.2], the equivalence of $I$ in the intersection is

$$
\int_{I}\left(1-i^{*} \gamma_{1}\right)^{12} c\left(\mathscr{N}_{I / X}\right)^{-1}
$$

Hence the number of twisted cubics intersecting 12 general lines in $\mathbf{P}^{3}$ is

$$
\int_{X}\left(-\gamma_{1}\right)^{12}-\int_{I}\left(1-i^{*} \gamma_{1}\right)^{12} c\left(\mathcal{N}_{I / X}\right)^{-1}=119020-38860=80160 .
$$

which agrees with the number found by Schubert [19, p. 179].

We turn now to the relative case. By the Leray-Hirsch theorem, we get a description of generators and relations of $A^{*}(\mathscr{X})$ as an $A^{*}(S)$-algebra. Recall the universal bundles $\mathscr{E}$ and $\mathscr{F}$ on $\mathscr{X}$, Again denote their Chern classes by $\gamma_{i}=c_{i}(\mathscr{E})$ and $\delta_{i}=c_{i}(\mathscr{F})$.

Proposition 7.6. $A^{*}(\mathscr{X})$ is generated as an $A^{*}(S)$-algebra by $\gamma_{1}, \gamma_{2}, \gamma_{3}$, and $\delta_{2}$. As an $A^{*}(S)$-module, it is isomorphic to $A^{*}(X) \otimes_{\mathbf{z}} A^{*}(S)$.

Proof. First note that each of $S, X$, and $\mathscr{X}$ have cellular decompositions [11, ex. 1.9.1], and hence the cycle maps give isomorphisms between Chow rings and integral singular cohomology. Hence it suffices to show that the classes above generate $H^{*}(\mathscr{X}, \mathrm{Z})$. But from prop. 7.1 we know that the monomials in these classes induce generators for the cohomology of the fibers of $\mathscr{X} \rightarrow S$. By the Leray-Hirsch theorem, the assertion of the proposition follows.

For relations, we imitate the description in the absolute case. The only real difference is that the vector space $V$ has been replaced by the vector bundle $\mathscr{V}$, and so the Chern classes of this bundle enter, twisting the relations a little bit. To be precise, let $m \in A^{1}(S)$ be the positive generator. Then $A^{*}(S)=\mathrm{Z}[m] / m^{5}$ and the total Chern class of $\mathscr{V}$ is

$$
c(\mathscr{V})=(1-m)^{-1}=1+m+m^{2}+m^{3}+m^{4} .
$$

Now put

$$
\tilde{R}=A^{*}(S)\left[e_{1}, e_{2}, e_{3}, f_{1}, f_{2}\right]=R \otimes_{\mathbf{z}} A^{*}(S)
$$


with the induced $W$-action. Let the maps $\tilde{\lambda}: \tilde{R} \rightarrow A^{*}(\mathbf{F})$ and $\tilde{\mu}: \tilde{R}^{W} \rightarrow A^{*}(\mathscr{X})$ be as the maps $\lambda$ and $\mu$, where $\tilde{\rho}: \mathbf{F} \rightarrow \mathscr{X}$ is the corresponding flag bundle associated to $\mathscr{E}$ and $\mathscr{F}$.

LEMMA 7.7. $\operatorname{Ker}(\tilde{\mu})$ contains the following three elements:

(i) $r_{0}=e_{1}+e_{2}+e_{3}-f_{1}-f_{2}$,

(ii) $r_{1}=\left(\sum_{j=0}^{4}\left(e_{1}-f_{1}\right)^{4-j} m^{j}\right)\left(\sum_{j=0}^{4}\left(e_{1}-f_{2}\right)^{4-j} m^{j}\right)$,

(iii) $\tilde{r}_{2}=\left(\sum_{j=0}^{4}\left(e_{1}-f_{2}\right)^{4-j} m^{j}\right)\left(\sum_{j=0}^{4}\left(e_{2}-f_{2}\right)^{4-j} m^{j}\right)$.

Proof. Reasoning as in the absolute case, $c_{8}\left(\rho^{*} \mathscr{F}^{\vee} \otimes \mathscr{E}^{1} \otimes \mathscr{V}\right)$ and $c_{8}\left(\mathscr{F}^{(1)^{\vee}} \otimes \mathscr{E}^{2} \otimes \mathscr{V}\right)$ vanish. But these are nothing but $\tilde{\mu}\left(\tilde{r}_{1}\right)$ and $\tilde{\mu}\left(\tilde{r}_{2}\right)$, respectively.

Put $\tilde{\mathbf{b}}=\sum_{i=0}^{2} c\left(\tilde{r}_{i}\right) \subseteq \tilde{R}^{W}$, where the $\tilde{r}_{i}$ are those of lemma 7.7. In view of lemma 7.2 we get

Proposition 7.8. Let $\tilde{\mathbf{b}}$ and $\tilde{\lambda}$ be as above. Then $\tilde{\mathbf{b}} \subseteq \operatorname{Ker}(\tilde{\lambda})$.

RemarK. Since $\tilde{\mathbf{b}}$ clearly reduces to $\mathbf{b}$ modulo $m$, it follows that $\left(\tilde{R}^{W} / \tilde{\mathbf{b}}\right) \otimes_{A^{*}(S)} \mathbf{Z}$ is isomorphic to $R^{W} / \mathbf{b}$. Since $\tilde{R}^{W} / \tilde{b}$ surjects onto the free $A^{*}(S)$-module $A^{*}(\mathscr{X})$, it follows that $\tilde{R}^{W} / \tilde{\mathbf{b}} \otimes_{\mathbf{z}} \mathrm{Q}$ is itself a free $A_{0}^{*}(S)$-module. Furthermore, since $\left(R^{W} / \mathbf{b}\right) \otimes_{\mathbf{z}} \mathrm{Q}=A_{0}^{*}(X)$, we deduce that $\left(\tilde{R}^{W} / \tilde{\mathbf{b}}\right) \otimes_{\mathbf{z}} \mathrm{Q}$ is isomorphic to $A_{\mathrm{Q}}^{*}(\mathscr{X})$. Note, however, that for the computation below we don't need more than prop. 7.8.

Proposition 7.9. Referring to prop. 6.2, we have

$\int_{\mathscr{X}} c\left(\mathscr{A}_{\mathscr{X}}\right)=256676750$ and $\int_{\mathscr{I}} c\left(i^{*} \mathscr{A}_{\mathscr{X}}\right) \cdot g_{*}\left(\frac{c\left(\mathscr{A}_{y}(-y)\right)-c\left(\mathscr{A}_{\mathscr{y}}\right)}{y c\left(\mathscr{A}_{\mathscr{y}}\right)}\right)=60529625$.

Proof. By computer symbolic computation.

In view of prop. 2.5 and prop. 6.2 , this gives

COROLLARY 7.10. The number of twisted cubics on a general quintic threefold is 317206375 .

In a spectacular computation using techniques from theoretical physics, Candelas et. al. [3] have found a conjectural procedure for computing the number $N_{d}$ of rational curves of any degree $d$ on a general quintic threefold. The number above coincides with their prediction for $\mathrm{N}_{3}$.

The computer files used in the computations in this paper are available from the authors upon request. 


\section{BIBLIOGRAPHY}

1. Dave Bayer and Michael Stillman, Macaulay, A system for computation in algebraic geometry and commutative algebra. Source and object code available for Unix and Macintosh computers. Contact the authors, or download from zariski.harvard.edu via anonymous $\mathrm{ftp}, 1990$.

2. B. Buchberger. Gröbner bases: An algorithmic method in polynomial ideal theory. In N. K. Bose, editor, Multidimensional Systems Theory, pages 184-232, Dordrecht-Boston-Lancaster, 1985. D. Reidel.

3. Philip Candelas, Xenia C. de la Ossa, Paul S. Green, and Linda Parkes, A pair of Calabi-Yau manifolds as an exactly soluble superconformal theory, Nuclear Phys. B, 359: 21-74, 1991.

4. Bruce W. Char, Keith O. Geddes, Gaston H. Gonnet, Michael B. Monagan, and Stephen M. Watt, Maple Reference Manual, Watcom Publications Ltd., Waterloo, Ontario, 5th edition, 1988.

5. Herbert Clemens, Some results about Abel-Jacobi mappings, In Phillip Griffiths, editor, Topics in Transcendental Algebraic Geometry, volume 106 of Annals of Mathematics Studies, pages 289-304, Princeton, New Jersey, 1984. Princeton University Press.

6. Herbert Clemens, Problems on 3-folds with trivial canonical bundle, In Birational Geometry of Algebraic Varieties, Open Problems, The Taniguchi Foundation, The XXIIIRD International Symposium Division of Mathematics, Katata, 1988.

7. L. Cremona, Note sur les cubiques gauches. J. Reine Angew. Math., 60 (1862), 188-192.

8. Jean-Marc Drezet, Fibrés exceptionnels et variétés de modules de faisceaux semi-stables sur $\mathrm{P}_{2}(\mathrm{C})$. J. Reine Angew. Math. 380, 14-58.

9. Geir Ellingsrud and Stein Arild Strømme, On the Chow ring of a geometric quotient, Ann. of Math., 130 (1989), 154-187.

10. Geir Ellingsrud, Ragni Piene, and Stein Arild Strømme, On the variety of nets of quadrics defining twisted cubic curves. In Franco Ghione, Christian Peskine, and Eduardo Sernesi, editors, Space Curves, Lecture Notes in Math. 1266, 1987.

11. William Fulton, Intersection Theory, Ergebnisse der Mathematik und ihrer Grenz-Gebiete, 3. Folge, Band 2. Springer-Verlag, Berlin-Heidelberg-New York, 1984.

12. Alexander Grothendieck, Sur quelques propriétés fondamentales en théorie des intersections. Séminaire Chevalley 2e année. Secr. Math. Paris, 1958.

13. Alexander Grothendieck, Techniques de construction et théorèmes d'existence en géométrie algébrique IV: Les schémas de Hilbert, Séminaire Bourbaki, 221, 1960/61.

14. Laurent Gruson, Robert Lazarsfeld, and Christian Peskine, On a theorem of Castelnuovo and the equations defining space curves. Invent. Math. 72 (1983), 491-506.

15. Sheldon Katz, On the finiteness of rational curves on quintic threefolds, Compositio Math. 60 (1986), 151-162.

16. David Mumford and John Fogarty, Geometric Invariant Theory, Number 34 in Ergebnisse der Mathematik und ihrer Grenz-Gebiete. Springer-Verlag, Berlin-Heidelberg-New York, 1982.

17. Ragni Piene and Michael Schlessinger, On the Hilbert scheme compactification of the space of twisted cubics. Amer. J. Math., 107 (1985), 761-774.

18. Th. Reye, Ueber lineare Systeme and Gewebe von Flächen zweiten Grades. J. für Math., 82 (1877), 54-83.

19. Hermann Schubert, Kalkül der Abzählende Geometrie, Verlag von B. G. Teubner, 1879. Repprinted by Springer-Verlag 1979, with an introduction by S. Kleiman. 